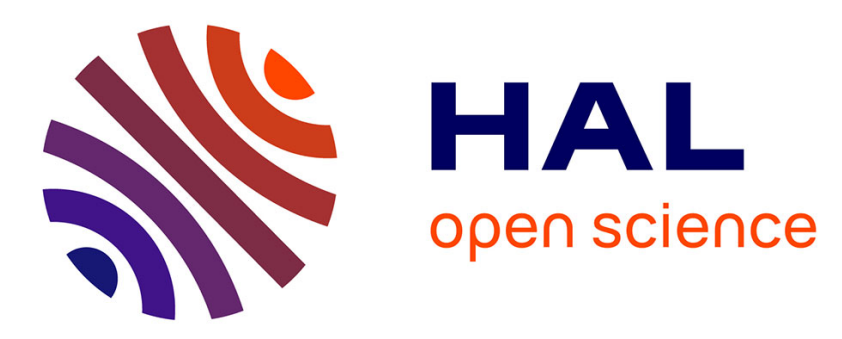

\title{
Manipulating dissipative soliton ensembles in passively mode-locked fiber lasers
}

François Sanchez, Philippe Grelu, Hervé Leblond, Andrey Komarov, Konstantin Komarov, Mohamed Salhi, Alioune Niang, Foued Amrani, C. Lecaplain, S. Chouli

\section{To cite this version:}

François Sanchez, Philippe Grelu, Hervé Leblond, Andrey Komarov, Konstantin Komarov, et al.. Manipulating dissipative soliton ensembles in passively mode-locked fiber lasers. Optical Fiber Technology, 2014, 20 (6), pp.562-574. 10.1016/j.yofte.2014.06.009 . hal-03198996

\section{HAL Id: hal-03198996 \\ https://univ-angers.hal.science/hal-03198996}

Submitted on 15 Apr 2021

HAL is a multi-disciplinary open access archive for the deposit and dissemination of scientific research documents, whether they are published or not. The documents may come from teaching and research institutions in France or abroad, or from public or private research centers.
L'archive ouverte pluridisciplinaire HAL, est destinée au dépôt et à la diffusion de documents scientifiques de niveau recherche, publiés ou non, émanant des établissements d'enseignement et de recherche français ou étrangers, des laboratoires publics ou privés. 
Invited Paper

\title{
Manipulating dissipative soliton ensembles in passively mode-locked fiber lasers
}

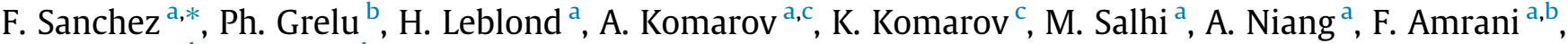 \\ C. Lecaplain ${ }^{\mathrm{b}}$, S. Chouli ${ }^{\mathrm{b}}$
}

a Laboratoire de Photonique d'Angers E.A. 4464, Université d'Angers, 2 Bd. Lavoisier, 49000 Angers, France

${ }^{\mathrm{b}}$ Laboratoire ICB, UMR CNRS 6303, Université de Bourgogne, 9 Av. A. Savary, BP 47870, 21078 Dijon Cedex, France

${ }^{\mathrm{c}}$ Institute of Automation and Electrometry, Russian Academy of Sciences, Acad. Koptyug Pr. 1, 630090 Novosibirsk, Russia

\section{A R T I C L E I N F O}

\section{Article history:}

Available online 16 July 2014

\section{Keywords:}

Fiber laser

Mode-locking

Ultrashort pulse

Soliton

Optical injection

\begin{abstract}
A B S T R A C T
We review our recent experimental and theoretical results addressing the dynamics of large numbers of solitons interacting in presence of a background in passively mode-locked erbium-doped fiber lasers. We first characterize experimentally the soliton rain complex dynamics, and then we focus on ordered soliton patterns. We report that, for suitable experimental parameters, a continuous wave can impose harmonic mode locking. Two levels of modeling for a mode-locked laser subjected to the external injection of a continuous wave are developed to support the latter observation. The first one is based on a scalar master equation, while the second one takes into account the mode-locking mechanism more accurately through a vectorial approach.
\end{abstract}

(ㄷ) 2014 Elsevier Inc. All rights reserved.

\section{Introduction}

Although the area of passively mode-locked fiber lasers has witnessed more than 20 years of development, fiber laser dynamics remains under intensive investigations, since there remain many challenges at both fundamental and applied levels. Numerous applications require the increase of the pulse energy. For that purpose, it is necessary to limit the dynamics to single pulse operation. Significant increase of the pulse energy has been realized by using parabolic pulses [1,2] and, more recently, all-normal-dispersion cavities $[3,4]$. Another area of intense investigation is the realization of frequency combs $[5,6]$, for which stable, multi-wavelength operation is needed in view of applications such as dense wavelength-division multiplexing [7] or optical metrology [8]. Highrepetition-rate fiber lasers naturally deliver such frequency comb distributions [9]. However, the challenge is to obtain a flat spectral distribution with a tailored wavelength separation and enhanced comb stability [10]. In the temporal domain, these performances correspond to accurately controlling the pulse distribution along the cavity and, consequently, the interactions between ultra-short pulses. At the fundamental level, passively mode-locked fiber lasers constitute a one-dimensional-propagation nonlinear system in which dissipative solitons naturally manifest [11]. Fiber lasers

\footnotetext{
* Corresponding author.

E-mail address: francois.sanchez@univ-angers.fr (F. Sanchez).
}

constitute an ideal platform to investigate the interaction between ultra-short pulses, especially in the anomalous dispersion regime, where numerous solitons can be simultaneously excited at high pumping power [12-14]. Various soliton patterns, analogous to the states of matter, have been observed, regardless of the given mode-locking mechanism, highlighting universal interaction properties [15-20]. The soliton distribution in a cavity roundtrip is a direct consequence of these interactions, which can be either repulsive or attractive, depending on time scales and cavity parameters involved. Dominantly attractive interactions are responsible for the formation of bound states, which are composed of identical solitons [21], and whose size culminates in large soliton crystals [22]. Harmonic mode locking (HML) is the consequence of a dominantly repulsive interaction, which was until recently attributed to the gain relaxation dynamics [23]. However, in many HML fiber laser experiments, a continuous wave (cw) component has been noticed in the optical spectrum, suggesting that this component could play an important role in the HML mechanism [24-27]. In addition, it was theoretically shown that a small cw component allowed controlling the nature and the strength of the soliton interaction [28]. This prediction together with the observation of the cw component in the HML led several teams to investigate the influence of an external cw on the soliton distribution in passively mode-locked fiber lasers. The basic idea is to determine if it is possible to control the soliton interaction through continuous external injection. Let us note that continuous optical injection has 
been used to efficiently stabilize a passively mode-locked quantum dot semiconductor laser [29,30]. Indeed, these lasers usually suffer from waveform instability at high pump power. It was demonstrated that the external $\mathrm{cw}$ component had a stabilizing effect together with spectral narrowing.

The paper is organized as follows. To give the flavor of complexity that is associated with multi-soliton dynamics, we develop in Section 2 the study of the soliton rain dynamics. In the latter, soliton pulses are continuously generated from an extended noisy background and drift until they reach a condensed phase comprising several aggregated solitons. This quasi-stationary dynamics, which was observed in several cavity configurations [32-34], can be triggered with an external cw signal [32]. By using high-bandwidth detection electronics, we here highlight the interaction between the drifting solitons and the condensed soliton phase. Section 3 relates the experimental testing of $\mathrm{cw}$ injection to achieve high-harmonic mode locking, in a high-power double-clad Er-doped fiber laser. Starting from an irregular initial soliton distribution, we show that, under specific injection conditions, the external laser can force the mode-locked laser to operate in the HML regime [35]. Theoretical and numerical modeling approaches are considered in Section 4. The first approach is based on a universal master equation [36] in which a driven cw term is added. It shows that, starting from different soliton patterns, the external injected term induces a motion of the solitons then leading to various distributions [37]. The second modeling approach is based on a vectorial model, and considers the interaction of few solitons submitted to an external continuous field. It is demonstrated that under specific resonance conditions, repulsive or attractive interactions occur between solitons leading to harmonic mode locking or soliton crystal patterns, respectively [38].

\section{Soliton rain}

A common understanding is that mode locking appears as an abrupt transition between a noisy continuous wave regime and a "clean" short-pulse laser operation. However, complex short pulse dynamics, from stationary to pulsating and chaotic ones, have been found in the vicinity of conventional mode-locked regimes [3942]. The concept of dissipative solitons provides a clearer understanding of the wide range of short pulse dynamics that can be accessed when the parameters of the laser system are varied [11]. In addition, mode locking has been identified to behave as a first-order phase transition, using statistical light mode dynamics [43]. These theoretical backgrounds help to understand the existence of partial mode locking, where mode-locked pulses coexist with a significant noisy or quasi-cw background. Soliton rain dynamics represents a stunning illustration of this situation, where soliton pulses and a noisy set of cw components, share the total cavity energy and interact in a dramatic quasi-stationary fashion [44].

This regime was found in a fiber ring laser cavity operated in the vicinity of conventional mode locking [32]. The laser cavity, sketched in Fig. 1(a), is built around a 2-m long erbium-doped fiber amplifier pumped by two 980-nm laser diodes with a maximal injected power of $800 \mathrm{~mW}$. Mode locking is achieved by using nonlinear polarization evolution along the fibers, followed by intensity discrimination by a polarizer. Tuning the fibered intracavity polarization controllers shapes the nonlinear transfer function. With such flexibility, numerous dynamical regimes become accessible besides standard mode locking, by simply altering the orientation of the polarization controllers, among which the soliton rain dynamics described below.

Propagation in the anomalous dispersion regime combined with an intense pumping power ensures multiple-pulse operation with, typically, 10-100 soliton pulses, which tend to aggregate into a sub-nanosecond pulse bunch. At the same time, a significant fraction of the energy of the cavity remains in the quasi-cw background. This combination of mode locking and background components can be seen in the optical spectrum (Fig. 1(b)), with the presence of a quasi-cw spike in addition to the resonant radiation waves symmetrically located with respect to the center of the spectrum [45]. Such mixing of field components is also apparent in the temporal domain (see Fig. 1(c)), which reveals an inhomogeneous background. Importantly, the fraction of the weakly coherent background could be gradually varied by tuning the cavity parameters, similar to the alteration of the proportions of mixed phases in the course of a first-order phase transition.

Soliton rain is a complex self-organized dynamics, which takes place among large numbers of solitons and a substantial background. It is characterized by the interactions between three main field components. The first component is a bunch of several tens of bound and jittering solitons dubbed as the condensed soliton phase, since it appears analogous to a liquid thermodynamical phase [15,32]. This component appears as the main peak on oscilloscope traces, whenever the individual soliton constituents are not temporally resolved. Like a liquid would evaporate, the condensed phase emits a large amount of radiation on one temporal side. That radiation indeed moves to shorter times due to the conjunction of anomalous dispersion and spectral asymmetry of the radiated waves [44]. This temporal asymmetry can be seen on magnified oscilloscope traces, and is also consistent with the analysis developed in Section 4.2. This radiation also superimposes with other preexisting $\mathrm{cw}$ modes, altogether producing a noisy, inhomogeneous background with large fluctuations. When fluctuations exceed a certain level, a new soliton is formed, such as a droplet formed from a vapor cloud, which then drifts back to the condensed phase at a nearly constant relative velocity, as can be seen in the stroboscopic plots shown on Fig. 1(c and d). Note that the relative drifting of pulses is a continuous slow motion performed one cavity roundtrip after another, whereas stroboscopic plots display a discontinuous sequence of recordings where two consecutive traces are separated by a $40 \mathrm{~ms}$ lapse of time, which corresponds to around $6 \times 10^{5}$ cavity rountrips. This slow drifting motion of isolated pulses towards the condensed phase operates at a relative velocity of the order of 10 meters per second, and is thus observable in real time on the oscilloscope. The whole scenario repeats in a quasi-stationary fashion, in the reference frame of the condensed phase that circulates round the cavity.

Since there is a noise threshold above fluctuations can be amplified to form the drifting solitons, it is possible to set the cavity operation - by lowering the pumping power, typically - below that threshold, and trigger the soliton rain by the injection of an external laser. Such triggering was experimentally demonstrated in Ref. [32].

Despite operation in the anomalous dispersion regime provides the easiest access to multiple pulse formation required in the soliton rain dynamics, similar dynamics were observed with fiber ring lasers operated in the normal dispersion regime $[33,46]$. In addition, soliton rain has been reported in a figure-of-eight fiber laser [34]. These observations tend to indicate that soliton rain, despite its complexity, represents a universal class of laser cavity dynamics.

Because there is a conjunction of internal motion and long-term quasi-stationarity in soliton rain dynamics, it is interesting to investigate the exchange of energy between field components, keeping aware that the whole system is not conservative, but dissipative with a constant supply from the pumping source. The noisy cw background energy seeds newly formed solitons, and then these drifting solitons transfer energy to the condensed 

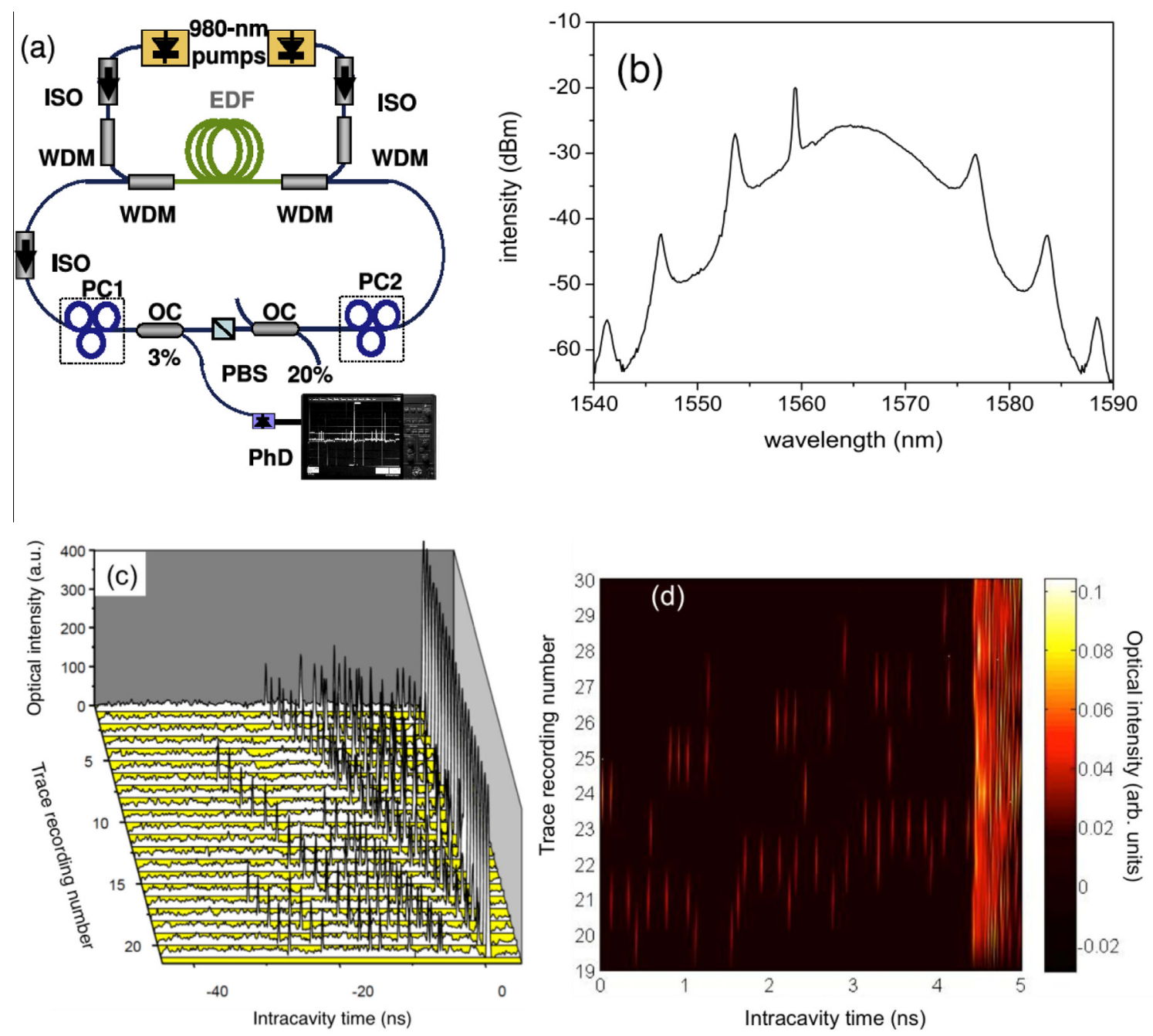

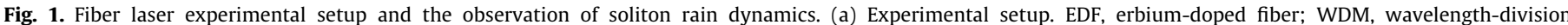

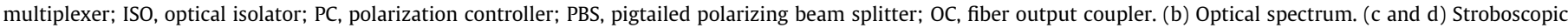

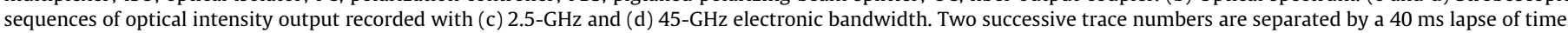

soliton phase. In order for the latter not to grow indefinitely, the radiation of the condensed soliton phase should be an important part of the whole soliton rain dynamics. In this article, we provide a better insight of soliton rain dynamics by analyzing collision and radiation processes using real-time recordings with a temporal resolution of $25 \mathrm{ps}$.

A temporal close-up view of the soliton rain dynamics is provided within Fig. 2, which consists of successive recordings of oscilloscope traces. The time between two successive oscilloscope traces is around $45 \mathrm{~ms}$. Each trace monitors the real-time optical intensity at the laser cavity output, through a $45-\mathrm{GHz}$ real-time sampling oscilloscope, whose electric signal is recorded via a 45 $\mathrm{GHz}$ photodiode. We obtain a partial resolution of the condensed phase, while capturing the incoming soliton pulses. Indeed, at a given time, few soliton pulses inside the condensed phase are sufficiently far from their neighbors to be temporally resolved. Here, the temporal duration of the condensed phase is around $900 \mathrm{ps}$. The number of solitons in the condensed phase can be calculated on the $45-\mathrm{GHz}$ real-time recording from the ratio between the energy of the condensed phase and that of an isolated soliton. In the present case, we start in Fig. 2(a) with 8 drifting solitons and 16 solitons in the condensed phase. The drift velocity of the isolated solitons relative to the condensed phase is around 6 meters per second. In Fig. 2(b), the collision between the two first drifting solitons and the condensed phase has occurred, so that the number of solitons amounts to 18 in the condensed phase. In Fig. 2(c), the last six drifting solitons have merged with the condensed phase, which tots up 24 solitons. This confirms that, at this stage, the 8 drifting solitons have transferred their energy to the condensed soliton phase. Subsequently, the number of solitons is progressively reduced to 20 (see Fig. 2(d)), then 18 (see Fig. 2(e)), which implicates the dissipation of solitons through radiation, since no soliton escape events are detected. In terms of dynamics and energy flows, we get at this stage a rough picture of the interactions between the drifting solitons and the condensed phase. We can follow the energy transfer to the condensed phase followed by its radiation. That clear picture of soliton rain dynamics is consistent with the fact that the size of the condensed phase remains constant on average. The arrival of new solitons causes, through collective rearrangements inside the condensed phase, the dissipation of a similar number of solitons. This dissipation in turn produces additional radiation and dispersive waves that contribute to the background, so that the cycle can be repeated.

Modeling soliton rain dynamics, which represents a daunting task due to the extent of temporal scales and multiple interaction processes involved, remains to be undertaken. We nevertheless hope that these latest experimental observations, by clarifying the collision-radiation process around the condensed soliton 

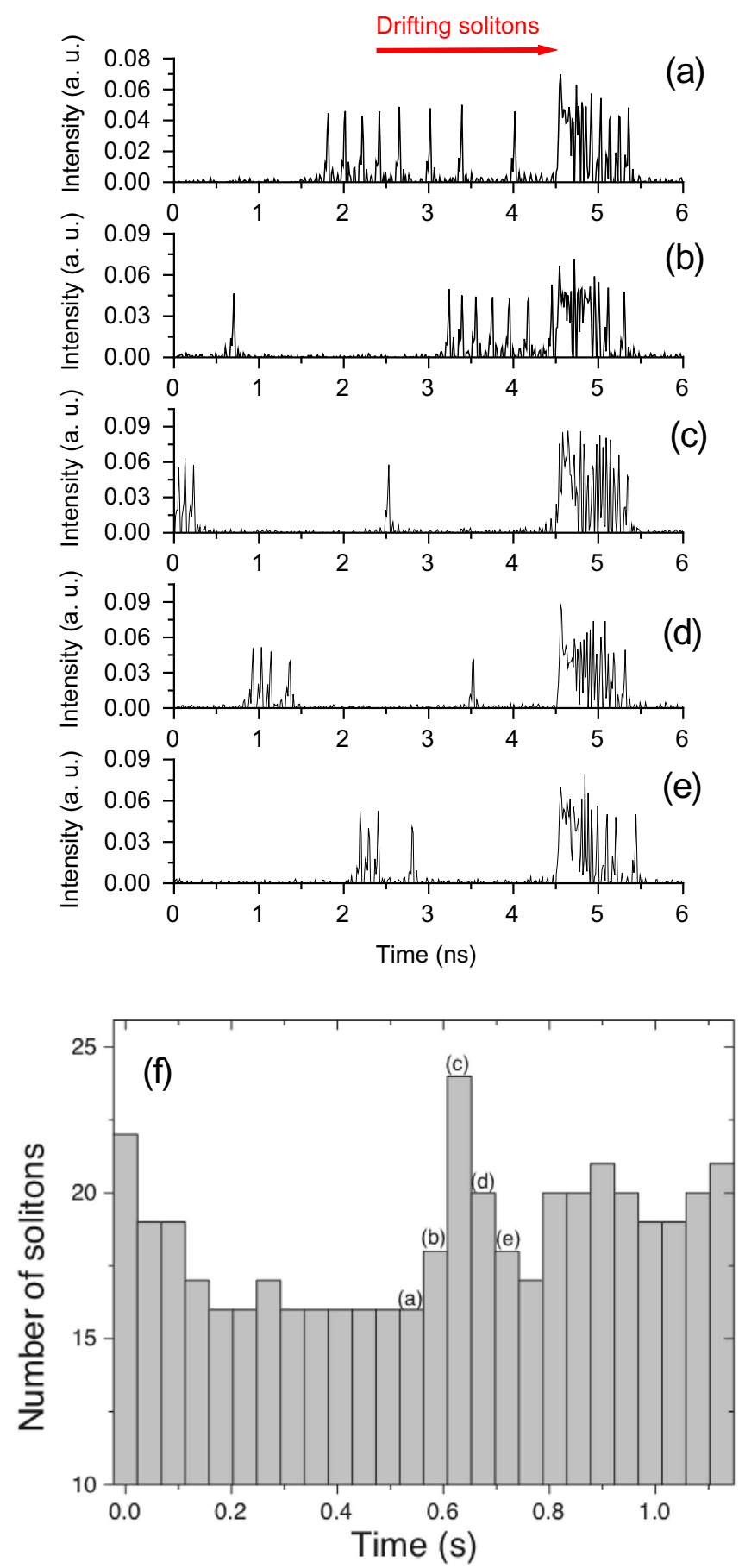

Fig. 2. Temporal close-up view of the drifting solitons impinging on the condensed soliton phase. (a)-(e) Oscilloscope traces displaying the optical intensity, recorded with a $45-\mathrm{GHz}$ electronic bandwidth. Between adjacent traces, the time interval is $40 \mathrm{~ms}$. (f) Evolution of the number of solitons in the condensed soliton phase highlighting successive absorption and radiation processes.

phase, will stimulate such modeling, which could be envisaged piece-wise, as a sequence of linked dynamical events.

\section{Harmonic mode locking through external injection}

In this section, we investigate experimentally the role of an external cw component on the passive harmonic mode-locking operation. A first study of this issue was performed in Ref. [47], where it was established that the external cw did not disturb the
HML regime significantly. This was probably due to the low power of the external injection source. Clear evidence on the influence of a cw wave on soliton interaction has been reported in Ref. [35]. We give hereafter the main features on this subject.

\subsection{Experimental setup and procedure}

The experimental setup is schematically represented in Fig. 3 $[27,35]$. It is an all-fiber unidirectional ring cavity using a doubleclad Er:Yb $10 \mathrm{~W}$ fiber amplifier operating at $\lambda=1.55 \mu \mathrm{m}$ specially designed for our experiments and manufactured by Keopsys. The double-clad fiber (DCF) is $5 \mathrm{~m}$ long and has a second order group velocity dispersion $\beta_{2}^{D C F}=-0.021 \mathrm{ps}^{2} / \mathrm{m}$. The inner clad has an octagonal shape with a diameter of $130 \mu \mathrm{m}$ and the fiber core diameter is $12 \mu \mathrm{m}$. It is pumped at $980 \mathrm{~nm}$ with several laser diodes injected with the v-groove technique. The maximum available pumping power is up to $40 \mathrm{~W}$, which ensures a total output power of amplified spontaneous emission up to $10 \mathrm{~W}$. The two fiber ends of the double-clad fiber are spliced to pieces of standard single-mode fibers (SMF28). The fibers DCF and SMF28 operate in the anomalous dispersion regime. A piece of dispersion-shifted fiber $\left(\beta_{2}^{D C F}=0.14 \mathrm{ps}^{2} / \mathrm{m}\right)$ is added to control the total cavity dispersion. To favor multiple-pulse mode locking, the total dispersion is set in the anomalous regime with $\beta_{2}^{D C F} L=-0.16 \mathrm{ps}^{2}$, with a total cavity length of $L=32.5 \mathrm{~m}$ corresponding to a round trip time of $162.4 \mathrm{~ns}$ and to a free spectral range of $6.16 \mathrm{MHz}$. Mode-locking is obtained thanks to the nonlinear polarization evolution technique based on nonlinear losses resulting from a polarizing isolator placed between two fibered polarization controllers [31]. As usual, the adjustment of the polarization controllers allows obtaining a large variety of soliton distributions [15]. When the main laser is mode-locked, the spectrum spans from $1540 \mathrm{~nm}$ to $1585 \mathrm{~nm}$. In the following the pumping power of the principal laser is fixed to $10 \mathrm{~W}$ which corresponds to about $2 \mathrm{~W}$ of average circulating power at $1.55 \mu \mathrm{m}$ and to $200 \mathrm{~mW}$ of average output power.

A high-speed photodetector (TIA-1200) connected to a fast oscilloscope (Tektronix TDS 6124C, $12 \mathrm{GHz}, 40 \mathrm{GSa}$ ) allows characterizing the temporal evolution of the signal. The spectral properties are analyzed with an optical spectrum analyzer (Anritsu MS 9710C) and the pulse duration is measured with an optical autocorrelator with a scanning range of \pm 100 ps (Femtochrome FR-103 XL). An electronic spectrum analyzer (Rohde \& Schwarz FSP Spectrum Analyzer $9 \mathrm{kHz}-13.6 \mathrm{GHz}$ ) is used to characterize the radio frequency spectrum of the laser.

The external continuous wave is provided by a homemade cw tunable fiber laser [48] and is launched with a 50/50 coupler to

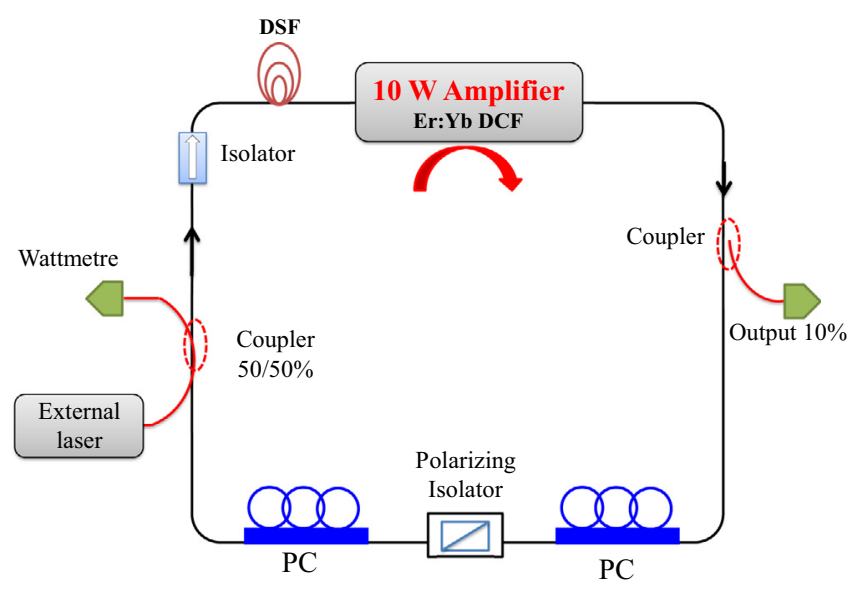

Fig. 3. Experimental setup. 
the principal cavity with a signal power that can be varied up to $800 \mathrm{~mW}$ (400 mW injected in the principal laser cavity). The external laser is tunable from $1530 \mathrm{~nm}$ to $1560 \mathrm{~nm}$ and its linewidth is about $1 \mathrm{~nm}$.

For injection experiments, we processed as follows. First, the principal laser is switched on and the polarization controllers are adjusted to obtain some soliton pattern. While this pattern is stable, we switch on the external laser and then increase its wavelength starting from $1530 \mathrm{~nm}$. Its influence on the operating regime of the principal laser is studied. Reversibility of the phenomena is checked in different ways. Firstly, the wavelength of the external laser is decreased to retrieve its initial value and secondly, we reduce the injected power and switch off the injection laser. The initial soliton distribution is restored in cases reported in Ref. [35].

\subsection{Experimental results}

With a suitable adjustment of the polarization controllers, we obtain the soliton pattern represented in Fig. 4. It consists in a set of well-separated soliton packets that do not have relative motions in the reference frame. Each packet contains different numbers of solitons and repeats from round-trip to round-trip. Additional insight is obtained from the optical spectrum shown in Fig. 5. The spectrum exhibits a modulation that is characteristic of a constant phase relation between the solitons, thus suggesting that the trains of solitons contain bound states [21], or soliton crystals [22], depending on the number of solitons involved in a given sequence. The spectral period is $0.2 \mathrm{~nm}$, which corresponds to a temporal separation of 41 ps. This value is confirmed by the autocorrelation trace given in Fig. 6, which shows that solitons are equidistant, and, because there is no pedestal, they are not in relative motion. In addition, the nearly triangular envelope indicates a regular soliton distribution in bound states. This initial soliton state is therefore a superposition of soliton crystals similar to those reported in Ref. [15]. The total number of solitons $N$ along the cavity can be estimated by dividing the total extent of the condensate phase $\Delta T_{c p}$ by the average delay between two solitons $\Delta \tau_{\text {sol }}$. The total extent of the condensate phases deduced from Fig. 4 is $\Delta T_{c p}=61 \mathrm{~ns}$. We assume that the average delay between neighboring solitons is $\Delta \tau_{\text {sol }} \cong 41 \mathrm{ps}$ in agreement with the autocorrelation trace. The total number of solitons is therefore $N=\Delta T_{c p} / \Delta \tau_{\text {sol }} \cong 1500$. Assuming a secant hyperbolic pulse shape, the width of the central peak of Fig. 6 leads to pulse duration of about 1 ps.

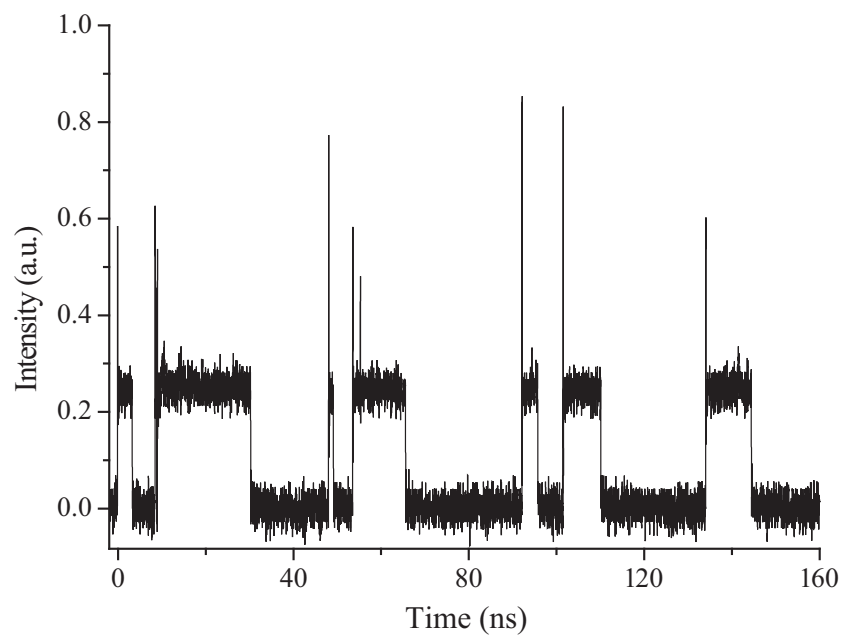

Fig. 4. Initial soliton distribution.

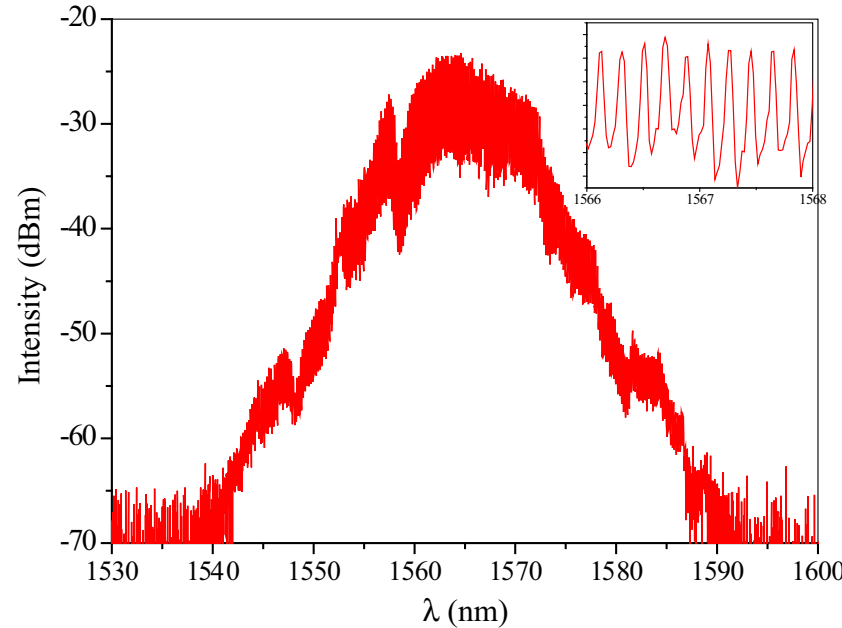

Fig. 5. Optical spectrum corresponding to data of Fig. 4. The inset displays a magnification.

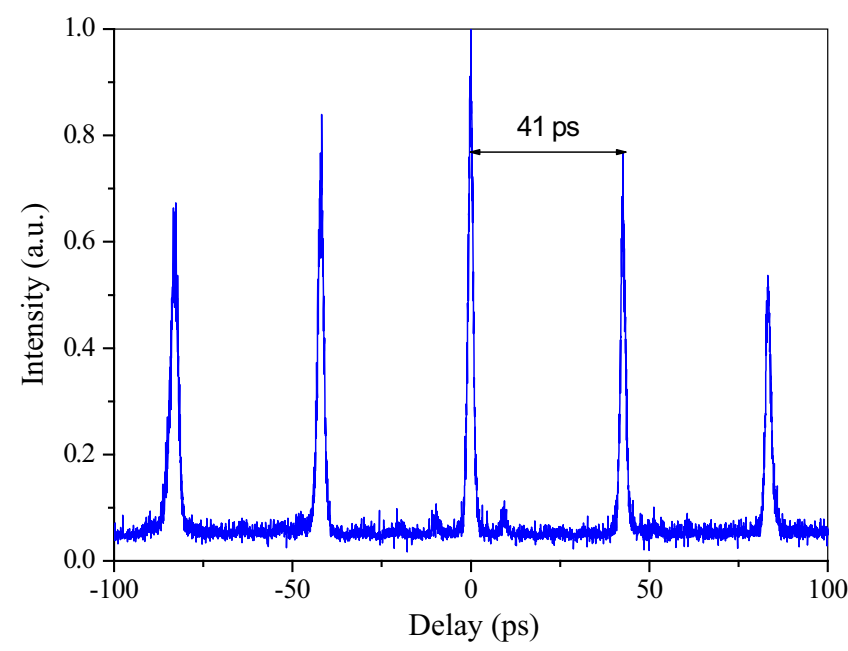

Fig. 6. Autocorrelation trace of the initial soliton distribution.

We then switched on the external laser with a start wavelength $\lambda_{\text {ext }}=1530 \mathrm{~nm}$ and with an injected power of $110 \mathrm{~mW}$ into the main laser cavity. The wavelength is then tuned towards longer values. While $\lambda_{\text {ext }}$ enters the spectrum of the principal laser, the spectral modulation decreases, revealing that the cw component significantly modifies soliton interactions until it breaks the strong coupling of the solitons in a bound state [49]. In the temporal domain, the soliton packets become unstable: they move and collide to form larger condensed phases without evident internal order. While $\lambda_{\text {ext }}$ falls in the spectrum of the principal laser, pulses get loose from the condensed phases and span over the whole cavity. Solitons move like in a soliton gas and when $\lambda_{\text {ext }}$ moves toward the center of the optical spectrum of the principal laser, the relative motion of the solitons decreases. When $\lambda_{\text {ext }}=1552 \mathrm{~nm}$, solitons stay at rest in the moving frame, nearly identical and equidistant as shown in Fig. 7. The optical spectrum given in Fig. 8 shows that there is no spectral modulation and, in addition to the cw component due to the injected laser $\left(\lambda_{\text {ext }}\right)$, there is an additional cw component generated by the principal laser $\left(\lambda_{\text {own }}\right)$ and characteristic of many passively HML fiber lasers [24-26]. The pulse duration, deduced from autocorrelation measurements, remains about 1 ps. HML is also pointed out through the radio-frequency spectrum of the recorded optical intensity, given in Fig. 9. The repetition rate 


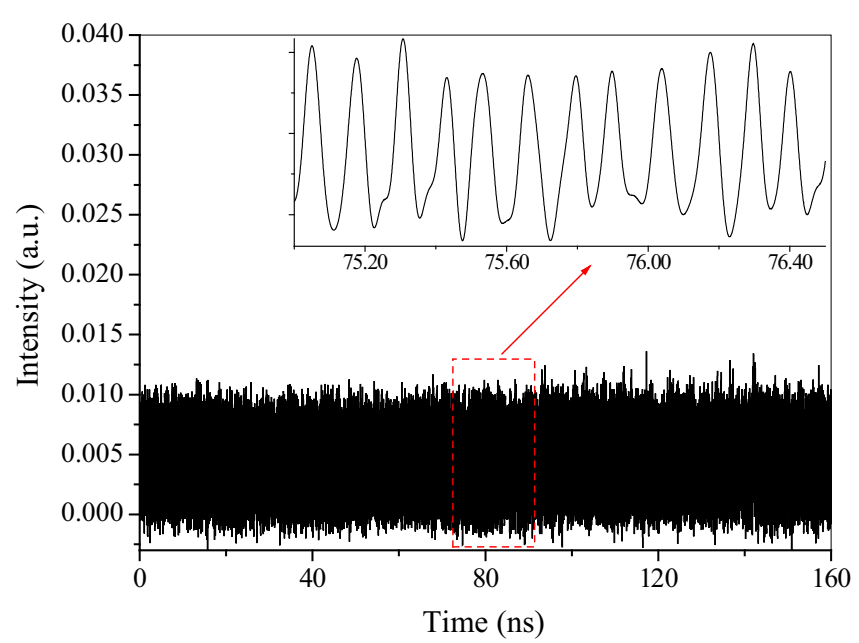

Fig. 7. Temporal trace of the harmonic mode-locking regime.

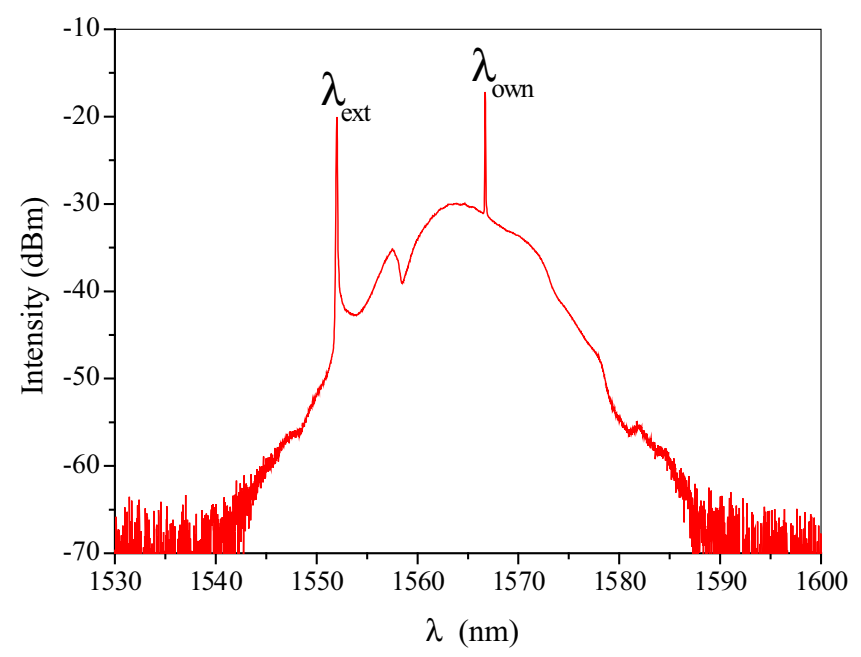

Fig. 8. Optical spectrum of the HML regime.

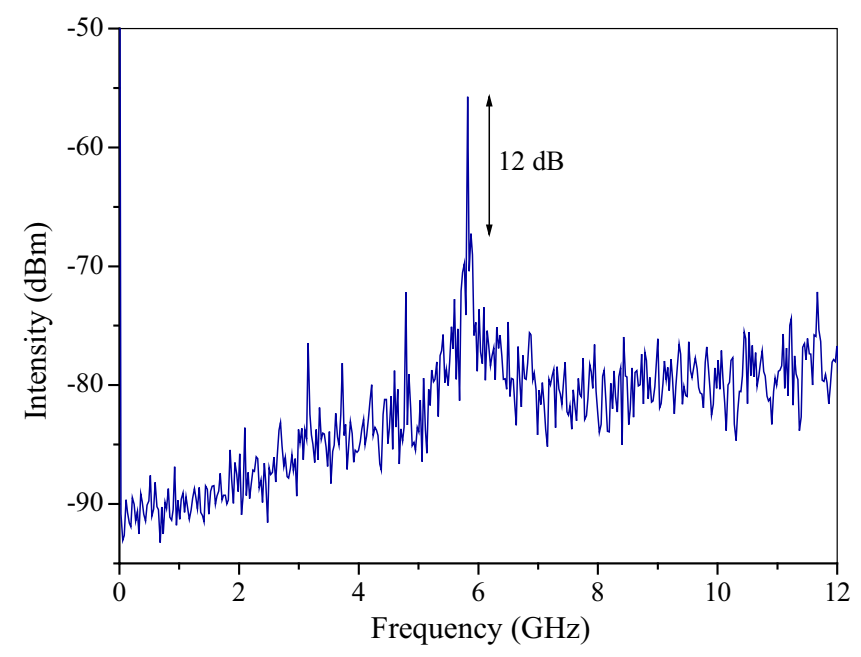

Fig. 9. Radio-frequency spectrum of the output intensity in the HML regime.

of the laser is precisely $5.82 \mathrm{GHz}$, which corresponds to the 945 th harmonics. The supermode suppression ratio is $12 \mathrm{~dB}$. The timing jitter and the amplitude fluctuations remain important and clearly visible in the zoom of Fig. 7. From the data series, the amplitude fluctuations are estimated to be about $18 \%$. Statistical analysis of the data series obtained with the delays between consecutive solitons gives a variance of about 58 ps. The variance provides an upper limit for the time jitter and is probably overestimated because it corresponds approximately to the temporal resolution of the oscilloscope.

The number of soliton is about 1000 . Thus, while the reorganization of the solitons along the cavity takes place, the number of pulses is reduced by around one third. The pulse duration remains practically constant of the order of $1 \mathrm{ps}$ and one can expect that the energy per pulse remains constant as a consequence of the soliton energy quantization. We have measured that the average output power of the principal laser decreases by an amount of $10 \%$ (about $20 \mathrm{~mW}$ ) under injected signal. This reduction of the output power is related to the modification of the nonlinear losses by the external resonant cw component. In itself this reduction does not explain the significant decrease of the number of pulses per cavity round-trip. There is still an amount of missing energy that is necessary contained in both the self-generated $\mathrm{cw}$ component and the amplified cw injected laser. To be more quantitative, the $200 \mathrm{~mW}$ of initial average output power is equally distributed between 1500 solitons. When the laser operates in the HML regime under external injection, the total output power becomes $180 \mathrm{~mW}$, which is divided between $135 \mathrm{~mW}$ equally shared among solitons, and an amount of $45 \mathrm{~mW}$ shared between the two cw components.

If $\lambda_{\text {ext }}$ is further increased, the same HML regime persists until $\lambda_{\text {ext }}=1553 \mathrm{~nm}$. For longer values of $\lambda_{\text {ext }}$, the HML regime is lost and we obtain a soliton gas where all solitons are in perpetual motion and fill the whole cavity. If $\lambda_{\text {ext }}$ is now decreased, we observe the reverse scenario without any hysteresis phenomena: first the regime becomes HML and finally the initial soliton crystals and bound states are restored. Hence, in this experiment the effect of the external cw component is fully reversible. We have also verified that for $\lambda_{\text {ext }}=1552 \mathrm{~nm}$, if the injected power is reduced, the HML regime persists in a wide range of injected powers. Indeed, HML occurs until the injected power reaches the lower value $50 \mathrm{~mW}$. Below this power, the initial soliton distribution is retrieved. If the injected power is increased again, the HML regime is restored.

We are convinced that the cw component takes a key role in the self-organization of the pulses. Indeed, the initial bound state is formed thanks to the equilibrium between attraction and short distance repulsion. While the cw external field is tuned within the optical spectrum of the principal laser, this equilibrium is broken through the emergence of a repulsive interaction. When the condensed phases are destroyed, the pulses move under the effect of a long-range repulsion leading to the final soliton distribution (HML regime). The fact that HML regime occurs for particular wavelengths of the injected laser suggests that some resonance effect occurs. This is in agreement with the preliminary theoretical results reported in Ref. [38].

\section{Theoretical approaches}

In this section we consider two complementary theoretical approaches, of increasing numerical complexity and accuracy, for the modeling of a passively mode-locked fiber laser submitted to a continuous external wave. The first one is a scalar distributed model based on a universal master equation [36], and the second one takes into account the precise mode-locking mechanism [13] through a vectorial approach, and includes piece-wise laser cavity sections and lumped polarization elements. A common point to both models is to consider a coherent interaction between an intracavity $\mathrm{cw}$ and a soliton inside the laser cavity and this mutual 
coherence is essential to achieve locking phenomena. Concerning the level of coherence required from the injection laser in experiments, this point requires further investigation. However we note that the coherence of the intracavity field tends to improve, when compared to the coherence of the injection laser, owing to the effect of the gain medium through stimulated amplification.

\subsection{Scalar distributed model}

The complex Ginzburg-Landau (CGL) equation is one of the most relevant models for the description of mode-locked fiber lasers. The well-known master equation by Haus [50] is nothing but a stationary version of the CGL; fiber laser models of cubic and cubic-quintic CGL type have been derived from several specific setups [11,51-55]; further, extensive mathematical studies are devoted to this model [56]. Bound states of two and three soliton have been considered theoretically in the frame of the complex Ginzburg-Landau (CGL) equation [57-61].

The cubic-quintic CGL equation reads as

$\frac{\partial E}{\partial z}=\delta E+\left(\beta+i \frac{D}{2}\right) \frac{\partial^{2} E}{\partial t^{2}}+(\varepsilon+i) E|E|^{2}+(\mu+i v) E|E|^{4}$

where $E$ is the electric field amplitude, and the real normalized coefficients $\delta, \beta, D= \pm 1, \varepsilon, \mu$, and $v$ account for (algebraic) net linear gain, spectral gain bandwidth, dispersion, cubic and quintic nonlinear gain, and fourth-order nonlinear index, respectively. In a distributed laser model, the variable $z$ is the normalized number of round-trips, interpolated with a continuous variable to facilitate the analysis.

It must be noticed that free motion of solitons is not allowed within the CGL model. Indeed, the term $\beta \partial^{2} E / \partial t^{2}$, which accounts for finite spectral bandwidth of the gain, breaks the Galilean invariance of the CGL equation. More specifically, if $\beta$ is zero, and $E_{0}(z, t)$ is a solution to Eq. (1), then $E=E_{0}(z, t-w z) \exp i\left[D w t-\left(D w^{2} / 2\right) z\right]$ is another solution moving at inverse speed $w$. If $\beta \neq 0$, such a moving solution does not exist. It can be easily checked numerically that an initial data of the form of a one-soliton solution $E_{0}(z, t)$ of Eq. (1) put into motion by a phase factor $e^{i \Delta \omega_{1} t}$ with $\Delta \omega_{1}$ some frequency shift, suffers strong braking and quickly stops.

The external injected cw wave is taken into account by adding a source term to the CGL equation, which becomes

$$
\begin{aligned}
\frac{\partial E}{\partial z}= & \delta E+\left(\beta+i \frac{D}{2}\right) \frac{\partial^{2} E}{\partial t^{2}}+(\varepsilon+i) E|E|^{2}+(\mu+i v) E|E|^{4} \\
& +A \exp \left(-i \Delta \omega_{0} t\right)
\end{aligned}
$$

where $A$ is the amplitude of the injected cw and $\Delta \omega_{0}$ is the detuning. Eq. (2) is solved by means of a standard fourth-order RungeKutta algorithm in the Fourier domain, the nonlinear terms being evaluated at each sub-step by means of inverse and direct fast Fourier transforms. We use the parameters $\delta=-0.01, \beta=0.5, D=1$, $\varepsilon=0.4, \mu=-0.05$, and $v=0$, and vary $A$ and $\Delta \omega_{0}$. As Eqs. (1) and (2) breaks the Galilean invariance, but a soliton acquires some velocity depending on the amplitude $A$ and detuning $\Delta \omega_{0}$ of the injected cw. Starting from initial data of the form of a one-soliton solution to the CGL Eq. (1), and running the resolution of the propagation equation until $z=20000$, we evaluate the soliton relative velocity $w=\Delta t / \Delta z$, which represents the relative temporal displacement per unit of cavity roundtrip. The results are presented in Fig. 10. The velocity $w$ has a very low value, less than $10^{-4}$. At small detuning, and above some amplitude threshold of the cw component (slightly larger than $A=0.001$ at $\Delta \omega_{0}=0$ ), the one-soliton state becomes unstable, and an additional soliton may be formed. We limited the computation of velocity to the stability domain of the one-soliton. In a real laser cavity, this instability is counterbalanced by the saturation of the gain, which is not taken into account by the CGL model: the instability mentioned above is

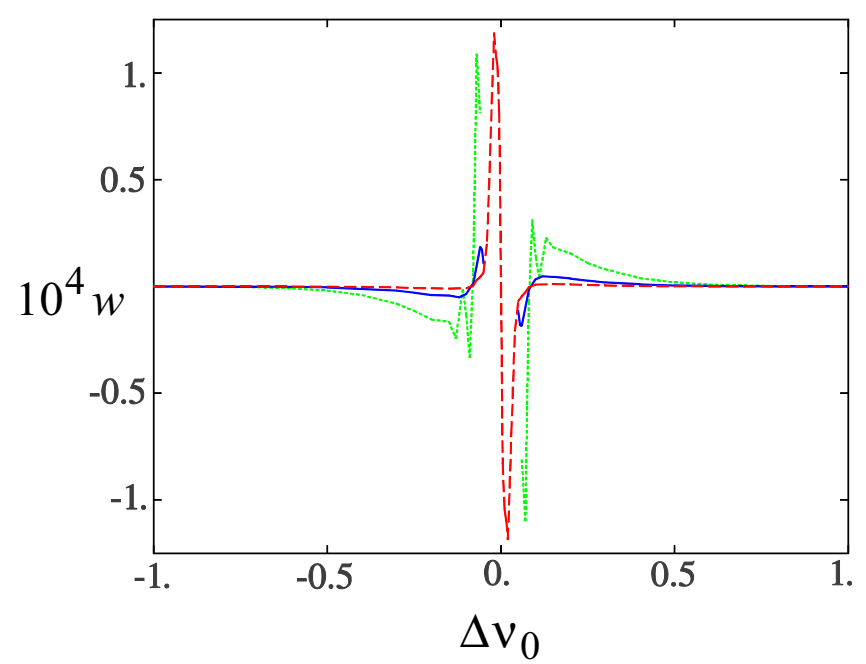

Fig. 10. Soliton inverse velocity $w=\Delta t / \Delta z$ against detuning $\Delta v_{0}=\Delta \omega_{0} / 2 \pi$ for a few values of the injected cw amplitude, namely $A=0.004$ (green dotted line), 0.002 (red dashed line), and 0.001 (blue solid line). (For interpretation of the references to colour in this figure legend, the reader is referred to the web version of this article.)

thus a mathematical property of the CGL model, which does not correspond to the real laser behavior.

Let us now consider a large number of solitons. We still solve numerically Eq. (2), with the same fixed parameters as above, varying the injected cw amplitude $A$ and frequency shift $\Delta v_{0}=\Delta \omega_{0} / 2 \pi$, but now we use as initial condition a soliton crystal solution of Eq. (1) that fills the whole computation box, and record the long-time regime that is reached after some transient. This transient corresponds to the stage of development of the instability mentioned above, however, the soliton crystal may remain stable in the presence of the cw component with amplitude in the considered range (0.1-2), in contrast to the single soliton. The results do not depend on the sign of $\Delta v_{0}$; depending on its value, the pulse train may behave as a crystal (Fig. 11(a)), a liquid (Fig. 11(b)), or a gas (Fig. 11(c)), which all are computed for the same value of the injected $\mathrm{cw}$ amplitude $(A=1.5)$. Note that this value is quite high with respect to the one considered in the previous section.

The optical spectra computed by means of a fast Fourier transform of the field $E$ for the same three examples are given in Fig. 12, and the autocorrelation trace $\int|E(\tau-t) E(\tau)|^{2} d \tau$, averaged on ten numerical steps, is shown on Fig. 13. The various outcomes are shown versus the amplitude and detuning in Fig. 14. The soliton crystal remains if the amplitude is small or the detuning large; a gas is produced in the opposite case. Intermediate patterns form, of liquid or polycrystal type, in between.

Hence, in the frame of the CGL equation, the injection of a cw component allows controlling the soliton distribution and also allows, by tuning the frequency of the $\mathrm{cw}$, to change the multisoliton pattern, from soliton crystal to soliton gas, via soliton liquid and polycrystal.

Since it can transform the soliton crystal into soliton gas, we see that the presence of a cw component can induce the apparent "Brownian motion" of the latter. It was shown above that the velocity induced by the cw component entirely depends on it. However, at the high amplitudes of injected cw considered here, the nonlinear interaction between cw component and pulses is much more complex. In particular, one can expect that the amplitudes of the considered radiative waves vary all along the cavity. Since we have seen that a tiny variation of the cw component in either amplitude or frequency was able to change radically the soliton velocity, the variations of the radiative waves change the soliton in the same way, and the resulting changes are apparently 


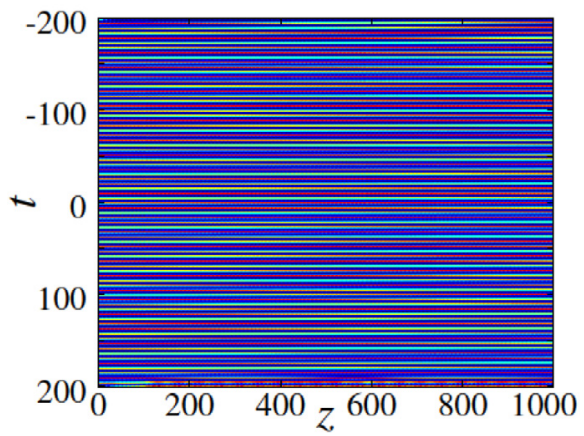

(a)

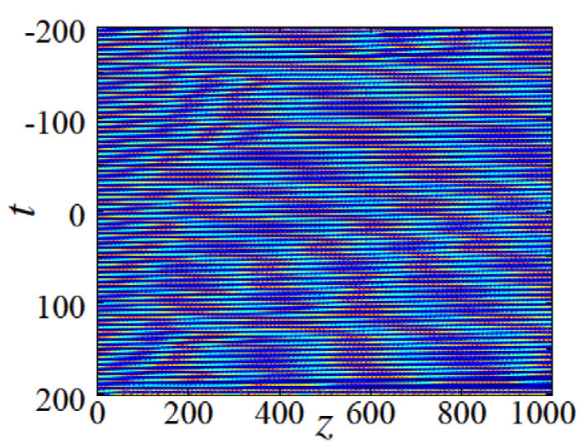

(b)

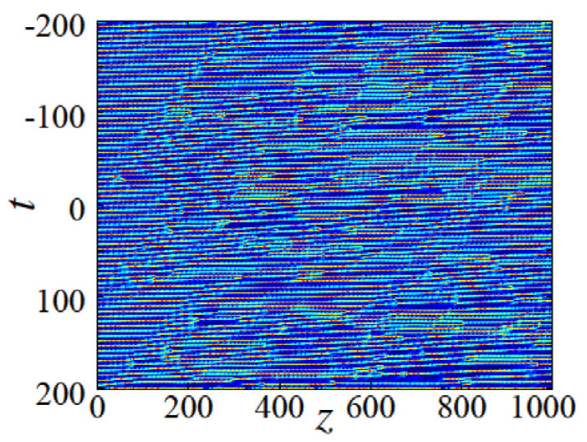

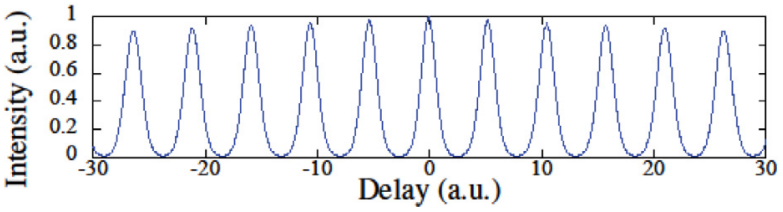

(a)

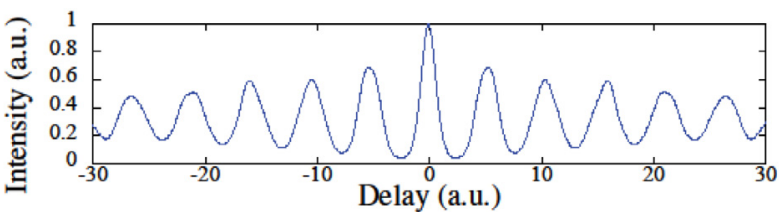

(b)

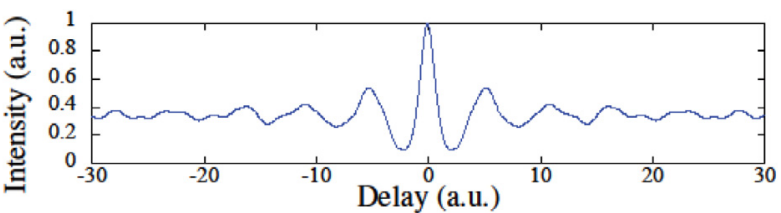

(c)

Fig. 13. Autocorrelation trace for the same three values of detuning as in Fig. 11. (a) Soliton crystal, (b) soliton liquid, and (c) soliton gas.

random. This is the explanation of the erratic motion in a soliton gas: radiative waves form in a quite erratic way. They induce soliton motion. The relation between the radiative waves and soliton velocity is single-valued but very complicated, so that its deterministic character does not prevent the general motion to appear as stochastic.

\subsection{Lumped vectorial model}

To develop our analysis of the role of an external optical injection on the interaction between solitons in a fiber laser with the nonlinear polarization rotation technique, we use now the vectorial model described by the following normalized equations $[13,16,38]$.

$$
\begin{aligned}
\frac{\partial E_{x}}{\partial \zeta}= & \left(D_{r}+i D_{i}\right) \frac{\partial^{2} E_{x}}{\partial \tau^{2}}+g E_{x}+i q\left(\left|E_{x}\right|^{2} E_{x}+A\left|E_{y}\right|^{2} E_{x}+B E_{y}^{2} E_{x}^{*}\right) \\
& +P \exp i(\delta \omega \tau-\delta \kappa \zeta)
\end{aligned}
$$

Fig. 11. Evolution of the temporal soliton pattern. (a) Soliton crysta
(b) soliton liquid for $\Delta v_{0}=0.9$, and (c) soliton gas for $\Delta v_{0}=0.8$.

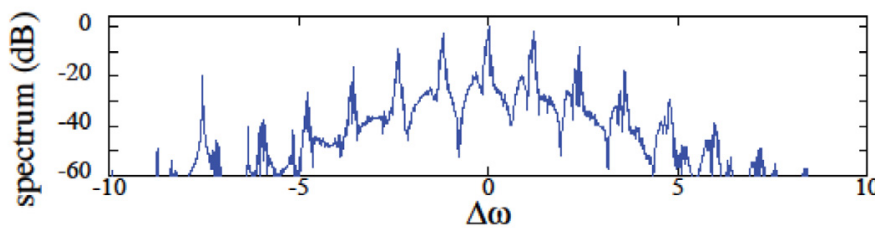

(a)

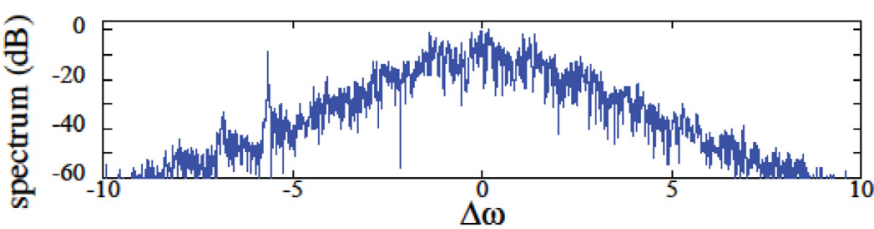

(b)

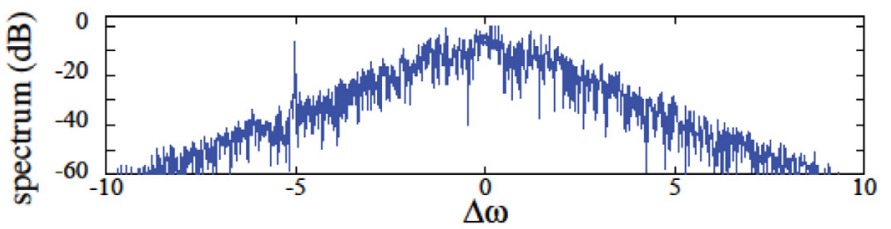

(c)

Fig. 12. Spectrum for the same three values of detuning as in Fig. 11. (a) Soliton crystal, (b) soliton liquid, and (c) soliton gas. 


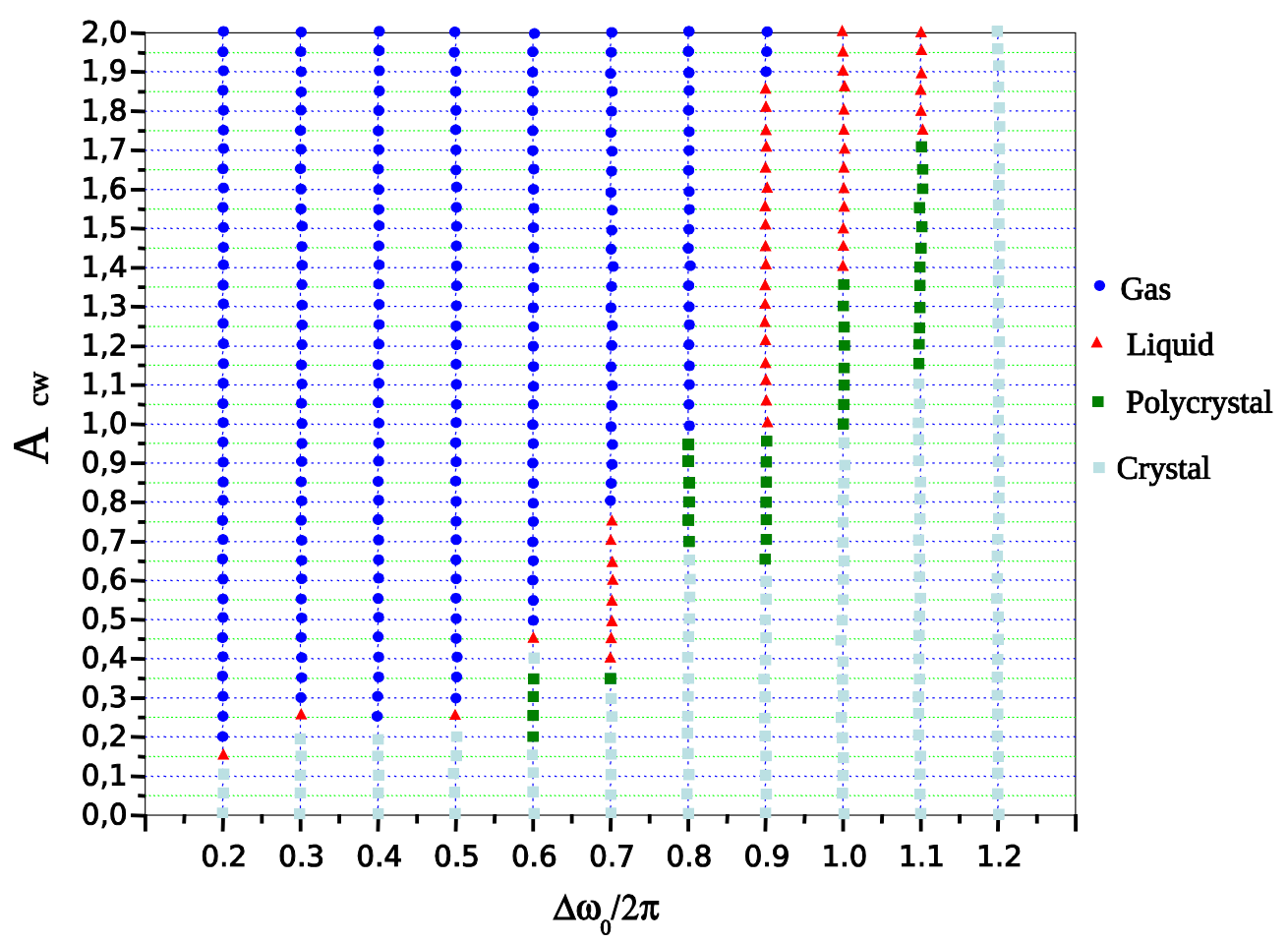

Fig. 14. The different regimes versus the detuning $\Delta \omega_{0}$ and the amplitude $A=A_{\mathrm{cw}}$ of the injected cw component.

$\frac{\partial E_{y}}{\partial \zeta}=\left(D_{r}+i D_{i}\right) \frac{\partial^{2} E_{y}}{\partial \tau^{2}}+g E_{y}+i q\left(\left|E_{y}\right|^{2} E_{y}+A\left|E_{x}\right|^{2} E_{y}+B E_{x}^{2} E_{y}^{*}\right)$

$E_{x}$ and $E_{y}$ are two orthogonal components of the electric field expressed in units $(\sqrt{\gamma L})^{-1}$ where $L(m)$ is the dimensional cavity length and $\gamma\left(W^{-1} \mathrm{~m}^{-1}\right)$ is the dimensional nonlinear refractive coefficient related to the nonlinear refractive index coefficient [13], $\zeta$ is the propagation distance (the number of passes of the radiation through the laser cavity), $\tau$ is the time coordinate in units $\delta t=\sqrt{\left|\beta_{2}\right| L / 2}$ where $\beta_{2}$ is the second-order group-velocity dispersion for the intracavity medium, $D_{r}$ is the frequency dispersion of the gain and the linear losses, $D_{i}$ is the frequency dispersion of the refractive index, $q$ is the normalized nonlinearity of the refractive index, $A=2 / 3, B=1 / 3$. The term $g$ describes the amplification in presence of saturation, namely $g=a /\left(1+b \int I d \tau\right)$, where the integration is carried out over the whole round-trip period, $a$ is the pumping parameter, $b$ is the saturation parameter and $I=\left|E_{x}\right|^{2}+\left|E_{y}\right|^{2}$. The last term in Eq. (3) describes the electric-dipole polarization of the intracavity medium that produces the monochromatic radiation inside the laser cavity. By this means, we model the injected radiation inside the laser cavity [38]. The parameters $\delta \omega$ and $\delta \kappa$ are determined by the frequency and wave vector of the external injected monochromatic radiation. Here $\delta \omega$ is the detuning of the carrier frequency of the external radiation from the center of the spectral gain band. The parameters $\delta \omega$ and $\delta \kappa$ are chosen so that the injected radiation coincides with the radiation of one of the longitudinal modes of the laser resonator. This implies that $\delta \omega$ and $\delta \kappa$ satisfy the dispersion relation $\delta \kappa=D_{i}(\delta \omega)^{2}$, which is obtained from Eq. (3). Consequently, in our numerical simulations we choose the frequency detuning in the form $\delta \omega=2 \pi K / l$, where the frequency parameter $K$ is an integer and $l$ is the length of the temporal computation box. The direction of a polarization of the linearly polarized injected cw coincides with the direction of the $x$-axis. Varying the parameters $P$ and $K$, we can change the intensity $I_{\mathrm{cw}}$ of the intracavity injected $\mathrm{cw}$ and its frequency detuning $\delta \omega$, respectively.
Eqs. (3), (4) describe the field evolution in the fiber of the unidirectional fiber ring laser shown in Fig. 15. The laser resonator contains a polarization control system including the following sequentially arranged components: a half-wave phase plate with orientation angle $\alpha_{2}$ with respect to the $x$-axis, a quarter-wave plate (orientation angle $\alpha_{1}$ ), a polarizing isolator (the passing axis is parallel to the $x$-axis), and a second quarter-wave plate (orientation angle $\alpha_{3}$ ) [13]. The polarization control system produces nonlinear losses that form ultrashort pulses in the laser resonator.

For our numerical simulation, the parameters of Eqs. (3) and (4) are chosen to be close to parameters of the passively mode-locked Er-doped laser investigated in Ref. [35] ( $a \approx 0.5, b=0.02, D_{r}=0.1$, $D_{i}=0.69, q=1, \alpha_{1}=0.2, \alpha_{2}=0.4, \alpha_{3}=-0.2$, and $\left.l \approx 164\right)$. Fig. 16 shows the characteristics of the intracavity radiation $\mathrm{cw}$ that is only due to the external optical injection. Here the amplification $g$ is less than linear losses due to a joint action of the polarizer and the phase plates. As a result, the intracavity radiation exists only because of the external injection. Varying the parameter $K$, we can change the frequency of the intracavity-injected cw. Changing the parameter $P$ we can change a value of its intensity $I_{\mathrm{cw}}$. The

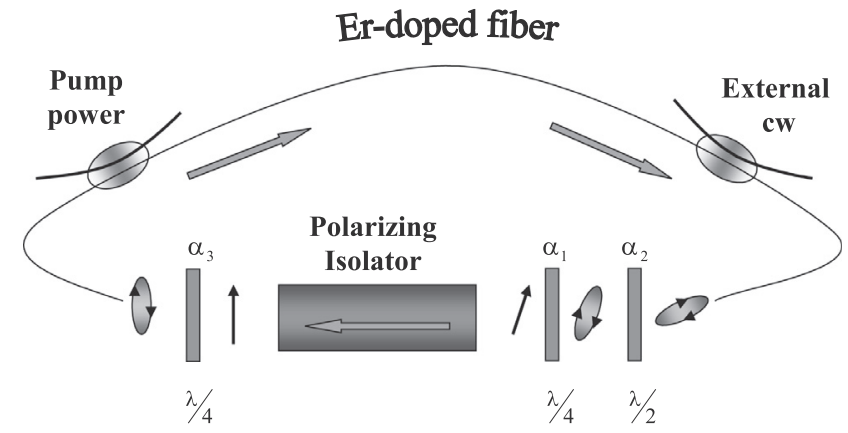

Fig. 15. Schematic representation of a passive mode-locked erbium-doped fiber laser based on nonlinear polarization rotation technique and submitted to an external cw injection. 

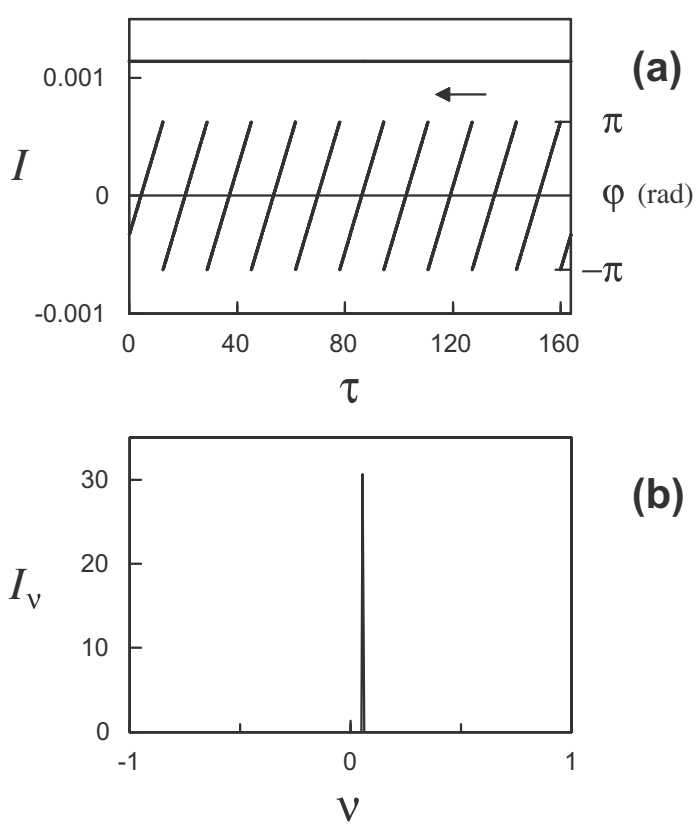

(b)

Fig. 16. (a) Distributions of the intensity $I(\tau)$ and phase $\varphi(\tau)$ of the intracavity radiation due to an external optical injection with a pumping $a$ below the threshold. (b) Spectral distribution of the radiation. Here $a=0.4, \alpha_{1}=0, \alpha_{2}=0.4, \alpha_{3}=0, K=10, P=0.1, l \approx 164$.

arrow in Fig. 16 shows a direction of a movement of points of the $\mathrm{cw}$ with a fixed phase. The total phase change of the $\mathrm{cw}$ on the length of the temporal computation box $l$ is equal to $2 \pi K$.

Fig. 17(a) shows the steady-state soliton that is formed in the laser cavity after a transient process. Here the injected external wave is absent $(P=0)$. The soliton has a pedestal with powerful wings due to dispersive waves. The soliton circulating in the laser cavity periodically experiences perturbations caused by lumped nonlinear losses and various intracavity components. After each perturbation, the soliton emits a dispersive wave. Constructive
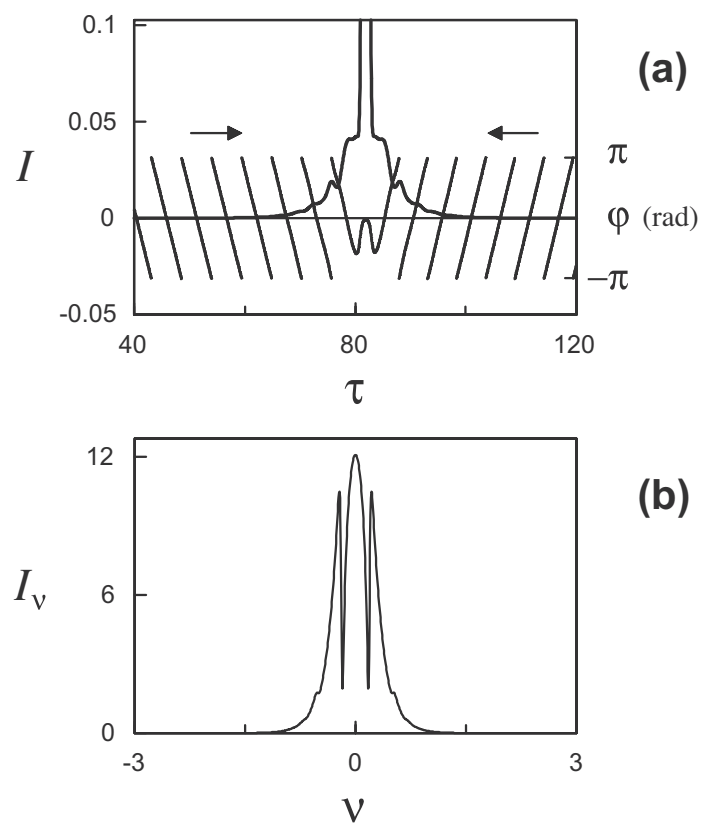

(b)

Fig. 17. (a) Distributions of the soliton intensity $I(\tau)$ and phase $\varphi(\tau)$ in the vicinity of the soliton pedestal without external optical injection $(P=0)$. (b) Spectral distribution of the soliton. interference between these waves forms powerful spectral sidebands (see Fig. 17(b)) and powerful extended soliton wings [62]. These wings result in long-range interaction, providing the formation of bound steady states of interacting solitons with a large binding energy [49]. As illustrated in Fig. 17(a), at the distant wings, the intensity $I(\tau)$ approaches zero. The radiation distribution $I(\tau)$ is repeated with a round-trip period $\delta \zeta=1$. The oscillations of the intracavity field with this period are due to the interaction of the soliton with the lumped intracavity elements. For the laser parameters used here, points with a certain fixed phase move to the center of the pulse. Arrows in Fig. 17(a) give the directions of this motion. The phase difference for any two fixed points of the field distribution does not change from one pass of the field through the laser resonator to the other.

Fig. 18 shows the steady-state distributions of the intensity $I(\tau)$, the phase $\varphi(\tau)$, and the spectrum of the intracavity radiation consisting of the steady-state soliton with the injected cw (here $P \neq 0$ ). At the distant wings of the soliton, the intensity $I(\tau)$ approaches the intensity $I_{\mathrm{cw}}$ of the injected $\mathrm{cw}$. The radiation distribution $I(\tau)$ is repeated with a round-trip period $\delta \zeta=1$. Here the phase difference for any two points of the field distribution is also repeated with this period that implies phase locking of the soliton and the intracavity-injected cw. As shown in Fig. 18(b), in the presented case, the frequency parameter $K$ of the injected cw corresponds to the peak of the right frequency sideband. This implies that the frequency parameter of the right soliton wing $K_{\mathrm{rw}}$ due to dispersive waves, coincides with the corresponding parameter of the injected $\mathrm{cw} K_{\mathrm{rw}}=K$. Accordingly, for the frequency parameter of the left soliton wing $K_{\mathrm{lw}}$, we have $K_{\mathrm{lw}}=-K$. The injected $\mathrm{cw}$ and the wave due to the right soliton wing form a destructive interference that decreases the resulting wave field at the right wing of the soliton (see Fig. 18(a)). This mechanism is also likely to be involved in the temporally asymmetric radiation of the condensed soliton phase depicted in Section 2 within the soliton rain dynamics. The injected cw and the wave due to the left soliton wing form a standing wave. Here it should be noted the following. First, the phases of the steady-state soliton and the cw increase linearly with increasing $\zeta$. When these rates of the phase increase coincide or are close
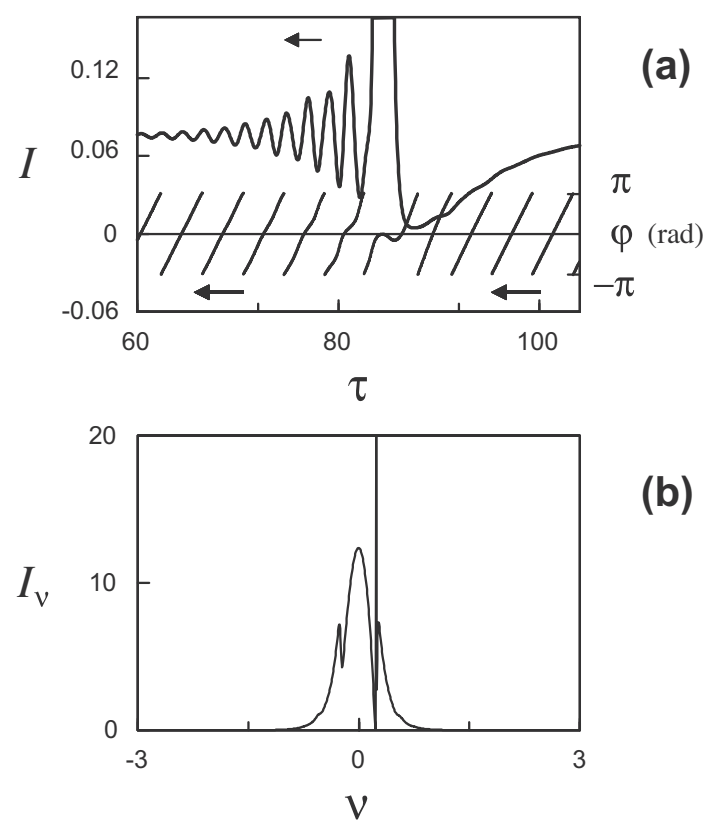

(b)

Fig. 18. (a) Distributions of the intensity $I(\tau)$ and phase $\varphi(\tau)$ of the field in the vicinity of the soliton pedestal in the case of an injected cw $(P \neq 0)$. (b) Spectral distribution of the resulting radiation. 


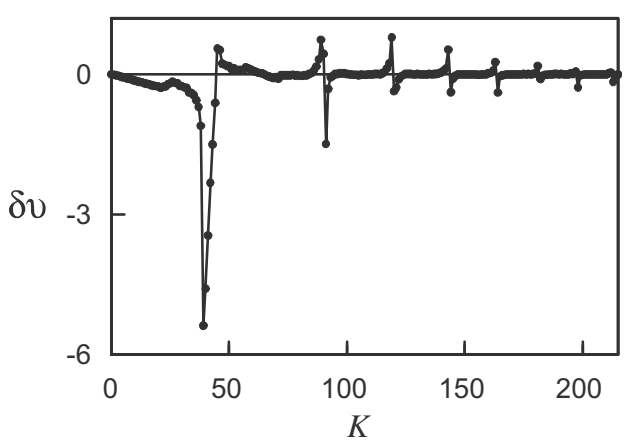

Fig. 19. Soliton velocity $\delta v$ vs the frequency parameter $K$ of the injected cw.

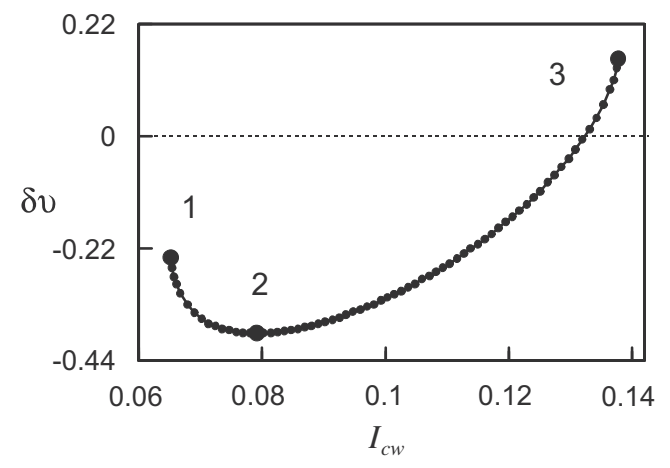

Fig. 20. Soliton velocity $\delta v$ vs the intensity $I_{\mathrm{cw}}$ of the injected cw. $K=40$.

to each other, resonant interaction between the soliton and the cw occurs. This interaction results in phase locking of the soliton and the cw. Such phase locking is realized in the case of Fig. 18. Second,

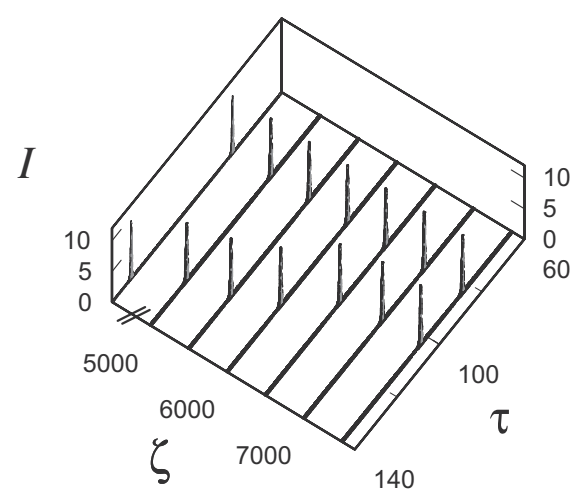

(a)

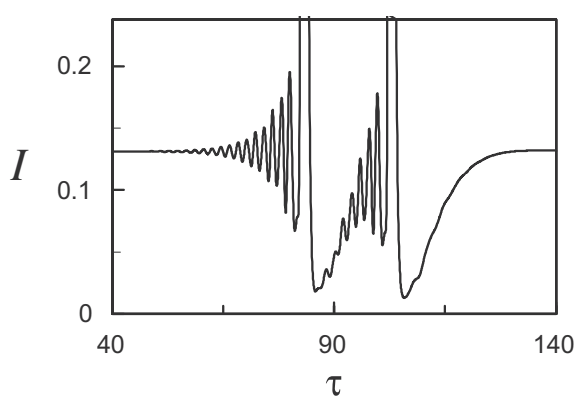

(b)

Fig. 21. Dependence of the intensity distribution $I(\tau)$ on the number of round trips $\zeta$ in a passive mode-locked laser with an injected cw. Regime of a soliton attraction. (a) Transient process. (b) Steady state (the scale is enlarged). $K=40, P=0.15$, $l \approx 164$.

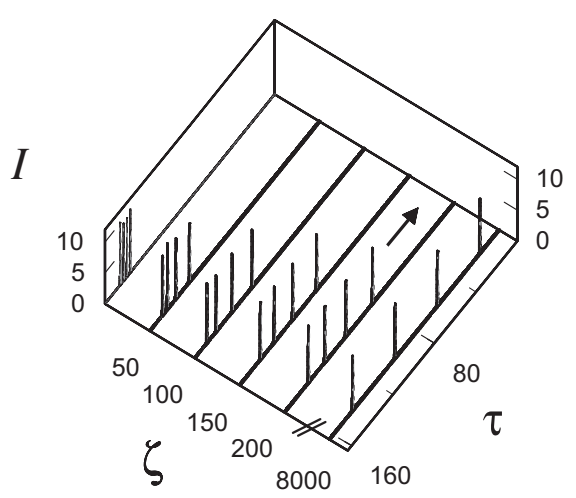

(a)

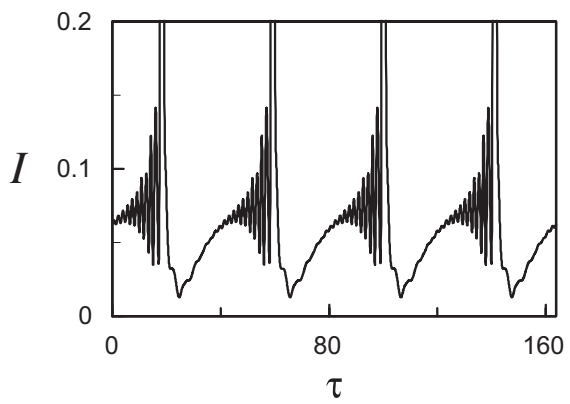

(b)

Fig. 22. Dependence of the intensity distribution $I(\tau)$ on the number of round trips $\zeta$ in a passive mode-locked laser with an injected cw. Regime of a soliton repulsion. (a) Transient process. (b) Steady state (the scale is enlarged). $K=40, P=0.1, l \approx 164$.

the powerful soliton wings and the powerful spectral sidebands are formed by dispersive waves for which the condition of the phase locking is fulfilled [38].

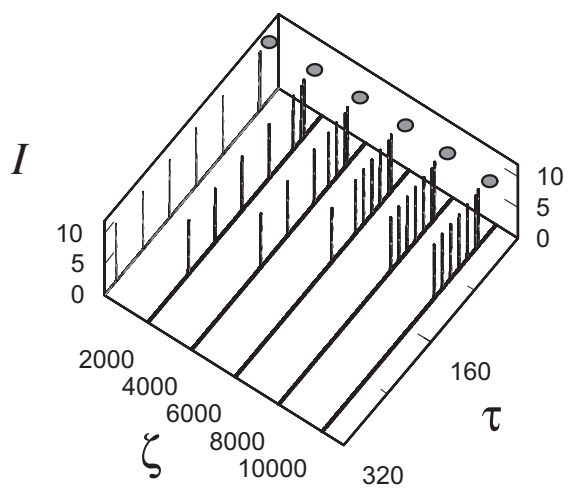

(a)

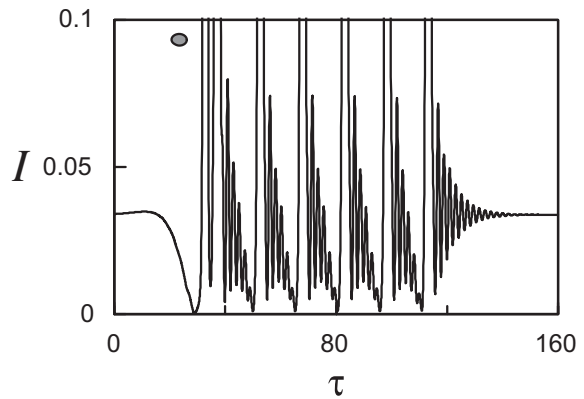

(b)

Fig. 23. Dependence of the intensity distribution $I(\tau)$ on the number of round trips $\zeta$ in a passive mode-locked laser with an injected cw. Regime of a soliton crystal. (a) Transient process. (b) Steady state (the scale is enlarged). The two-soliton molecule is marked by the grey circle. $K=76, P=0.09, l \approx 328$. 
Fig. 19 shows the dependence of the soliton velocity $\delta v=d \tau / d \zeta$ on the detuning parameter $K$ of the injected $\mathrm{cw}$. For the principal negative peak in the vicinity $K=40$, the change of the phase difference of the soliton and the cw over one round-trip period is equal to zero. Here the condition of the phase locking is fulfilled and the value $|\delta v|$ is large. Outside the phase-locking region, the phase difference for the soliton and the cw oscillates and the value $|\delta v|$ becomes small. For other peaks in Fig. 19 the change of the phase difference for the soliton and the cw over one round-trip period is a multiple of $2 \pi$.

Fig. 20 shows the dependence of the soliton velocity on the intensity $I_{\mathrm{cw}}$ of the injected cw. Phase locking of the soliton and the injected $\mathrm{cw}$ occurs in the intensity interval between points 1 and 3 . To the left of the point 1 , phase locking is absent. To the right of the point 3 , the injected cw destroys the single soliton operation. Between points 1 and 2 as well as between points 2 and 3 the dependence of $\delta v$ on $I_{\mathrm{cw}}$ is monotonic.

Fig. 21 demonstrates the attraction of two laser solitons. As a consequence of such interaction, after a transient process a stationary bound soliton is realized.

Fig. 22(a) shows a repulsion of laser solitons. After each pulse, the injected cw is attenuated because of its destructive interference with the right-wing dispersive waves. As a consequence, the resultant $\mathrm{cw}$ increases monotonically from left to right along the pulse train. The changing intensity of the resultant $\mathrm{cW}$ is between points 1 and 2 in Fig. 20, where the velocity of motion of pulses in the right direction increases monotonically with increasing intensity $I_{\mathrm{cw}}$. As a result, the velocity of the solitons motion in the right direction increases monotonically along the soliton train from left to right. This distribution of soliton velocities in the train results in an increase of inter-soliton spacing, that is, to repulsion of the solitons. In this case, after a transient process the harmonic passive mode locking (multi-pulse operation with equidistant pulses) is realized (see Fig. 22(b)). In the steady-state lasing regime, all pulses have the same phases at the points of their peak amplitudes. For the used laser parameters, the duration of the transient process is of the order of $10^{4}$ round-trip periods. In the steady-state regime of harmonic passive mode locking, the ratio of the inter-soliton interval to the soliton width is of the order of 100 . In the case of Fig. 21, the resultant cW is between points 2 and 3 of Fig. 20. Correspondingly an inter-soliton attraction is realized.

Fig. 23 demonstrates a formation of a soliton crystal through the light-induced drift of solitons in a laser cavity. The initial field is chosen in the form of a pair of strongly bound solitons and several individual solitons. The light-induced velocities of the soliton pair and an individual soliton are different. As a result, individual solitons drift toward the soliton pair, collide with it, stop and create a condensed soliton phase in the form of a soliton crystal.

\section{Conclusion}

In this paper we have presented an extensive review of the dynamics of a passively mode-locked fiber laser operated in the multi-pulse regime, when the interaction with a $\mathrm{cw}$ or quasi-cw background wave proves to be determinant. Hence, the basic idea was to address the important question on the possibility to manipulate soliton sequences in fiber lasers by controlling the level of the $\mathrm{cw}$ background. In a first part we have presented several experimental results demonstrating that the presence of a cw component has a strong influence in the soliton dynamics. Indeed, it allows either to control the appearance of drifting solitons in the soliton rain dynamics, or to generate a repulsive interaction between solitons in a stable condensed phase. Based on these experimental results, we have undertaken several theoretical approaches to describe the effect of an external cw on a given soliton distribution.
The first model is a scalar distributed model based on a universal master equation. Numerical simulations have shown that the external cw is able to induce a relative motion of solitons. Depending on both the frequency detuning and its amplitude, the cw leads to different soliton distributions such as a soliton crystal or a soliton liquid. However, this model does not reproduce harmonic mode-locking regimes such as those observed experimentally. This is in part due to the fact a fully distributed model lacks the essential cavity discretization effects, and the associated resonant interaction processes between solitons and radiated dispersive waves. To circumvent these limitations, we have constructed a second model closer to the realistic fiber laser case. It includes the vector properties of the electromagnetic field together with the exact mode-locking mechanism, including the lumped wave plate and polarizing components. Because of its larger parameter space and greater complexity, this model is restricted to the computation of the propagation and interaction of few solitons. Results have pointed out a resonance phenomenon between the spectral sidebands of the mode-locked laser and the frequency of the external cw. Depending on its amplitude and frequency, the cw is able to induce repulsive or attractive soliton interaction. As a consequence, the model is able to reproduce harmonic mode-locking regime involving repulsive and coherent soliton interaction. Complementing the traditional views of harmonic mode locking, which was thought to be essentially related to the slow gain relaxation dynamics [23], our description constitutes a significant progress towards a global understanding of multiple soliton dynamics, and the possibility of efficient manipulation of multi-soliton states inside of a laser cavity. More complex dynamics, as the soliton rain dynamics, still constitute considerable challenges for modeling due to the numerous processes and timescales involved. Still, advancing in that modeling direction will enable addressing a large class of fundamental multi-scale evolution problems as well as targeting optimized control routes for multisoliton states desirable in given applications, such as in high-repetition-rate all-fibered ultrashort pulse sources and stable frequency comb generation.

\section{Acknowledgments}

This work was supported by the Agence Nationale de la Recherche (Contract No. ANR-2010-BLANC-0417-01-SOLICRISTAL) and the Grant of Leading Scientific Schools of Russia No. 3753.2014.2.

\section{References}

[1] M.E. Fermann, V.I. Kruglov, B.C. Thomsen, J.M. Dudley, J.D. Harvey, Self-similar propagation and amplification of parabolic pulses in optical fibers, Phys. Rev. Lett. 84 (2000) 6010-6013.

[2] F.O. Idlay, J.R. Buckley, W.G. Clark, F.W. Wise, Self-similar evolution of parabolic pulses in lasers, Phys. Rev. Lett. 92 (2004) 213902.

[3] F.W. Wise, A. Chong, W.H. Renninger, High-energy femtosecond fiber lasers based on pulse propagation at normal dispersion, Laser Photonics Rev. 2 (2007) 58-73.

[4] A. Chong, J. Buckley, W. Renninger, F.W. Wise, All-normal dispersion femtosecond fiber laser, Opt. Express 14 (2006) 10095-10100.

[5] X. Feng, H.-Y. Tam, H. Liu, P.K.A. Wai, Multiwavelength erbium-doped fiber laser employing a nonlinear optical loop mirror, Opt. Commun. 268 (2006) $278-280$

[6] S.A.S. Melo, A.R. do Nascimento Jr., S. Arismar Cerqueria Jr., L.H.H. Carvalho, D.M. Pataca, J.C.R.F. Oliveira, H.L. Fragnito, Frequency comb expansion based on optical feedback, highly nonlinear and erbium-doped fibers, Opt. Commun. 312 (2014) 287-291.

[7] A.E.H. Oehler, S.C. Zeller, K.J. Weingarten, U. Keller, Broad multiwavelength source with $50 \mathrm{GHz}$ channel spacing for wavelength division multiplexing applications in the telecom C band, Opt. Lett. 33 (2008) 2158-2160.

[8] F.C. Cruz, Optical frequency combs generated by four-wave mixing in optical fibers for astrophysical spectrometer calibration and metrology, Opt. Express 16 (2008) 13267-13275.

[9] A. Komarov, A. Haboucha, F. Sanchez, Ultrahigh-repetition-rate bound-soliton harmonic passive mode-locked fiber lasers, Opt. Lett. 33 (2008) 2254-2256. 
[10] X. Feng, H.-Y. Tam, P.K.A. Wai, Stable and uniform multiwavelength erbiumdoped fiber laser using nonlinear polarization rotation, Opt. Express 14 (2006) 8205-8210.

[11] Ph. Grelu, N. Akhmediev, Dissipative solitons for mode-locked lasers, Nat. Photonics 6 (2012) 84-92.

[12] D.Y. Tang, L.M. Zhao, B. Zhao, A.Q. Liu, Mechanism of multisoliton formation and soliton energy quantization in passively mode-locked fiber laser, Phys. Rev. A 72 (2005) 043816.

[13] A. Komarov, H. Leblond, F. Sanchez, Multistability and hysteresis phenomena in passively mode-locked fibre lasers, Phys. Rev. A 71 (2005) 053809.

[14] X. Liu, Hysteresis phenomena and multipulse formation of a dissipative system in a passively mode-locked fiber laser, Phys. Rev. A 81 (2010) 023811.

[15] F. Amrani, A. Haboucha, M. Salhi, H. Leblond, A. Komarov, F. Sanchez, Dissipative solitons compounds in a fiber laser: analogy with the states of the matter, Appl. Phys. B 99 (2010) 107-114.

[16] D.Y. Tang, B. Zhao, L.M. Zhao, H.Y. Tam, Soliton interaction in a fiber ring laser, Phys. Rev. E 72 (2005) 016616.

[17] F. Amrani, M. Salhi, H. Leblond, F. Sanchez, Characterization of solitons compounds in a passively mode-locked high power fiber laser, Opt. Commun. 283 (2010) 5224-5230.

[18] F. Amrani, M. Salhi, H. Leblond, Ph. Grelu, F. Sanchez, Universal soliton pattern formation in passively mode-locked fiber lasers, Opt. Lett. 36 (2011) 15451547.

[19] A. Zaviyalov, R. Iliew, O. Egorov, F. Lederer, Multi-soliton complexes in modelocked fiber lasers, Appl. Phys. B 104 (2011) 513-521.

[20] Ph. Grelu, J.M. Soto-Crespo, Multisoliton states and pulse fragmentation in a passively mode-locked fibre laser, J. Opt. B: Quantum Semiclassical Opt. 6 (2004) S271-S278.

[21] Ph. Grelu, F. Belhache, F. Gutty, J.M. Soto-Crespo, Phase-locked soliton pairs in a stretched-pulsed fiber laser, Opt. Lett. 27 (2002) 966-968.

[22] A. Haboucha, H. Leblond, M. Salhi, A. Komarov, F. Sanchez, Analysis of soliton pattern formation in passively mode-locked fiber laser, Phys. Rev. A 78 (2008) 043806.

[23] J. Nathan Kutz, Mode-locked soliton lasers, SIAM Rev. 48 (2006) 629-978.

[24] F. Amrani, A. Haboucha, M. Salhi, H. Leblond, A. Komarov, Ph. Grelu, F. Sanchez, Passively mode-locked erbium-doped double-clad fiber laser operating in the 322th harmonic, Opt. Lett. 34 (2009) 2120-2122.

[25] G. Sobon, K. Krzempek, P. Kaczmarek, K.M. Abramski, M. Nikodem, 10 GHz passive harmonic mode-locking in $\mathrm{Er}-\mathrm{Yb}$ double-clad fiber laser, Opt. Commun. 284 (2011) 4203-4206.

[26] Z.X. Zhang, L. Zhan, X.X. Yang, S.Y. Luo, Y.X. Xia, Passive harmonically modelocked erbium-doped fiber laser with scalable repetition rate up to $1.2 \mathrm{GHz}$, Laser Phys. Lett. 4 (2007) 592-596.

[27] F. Amrani, A. Niang, M. Salhi, H. Leblond, F. Sanchez, Passive harmonic mode locking of soliton crystals, Opt. Lett. 36 (2011) 4239-4241.

[28] A. Komarov, K. Komarov, H. Leblond, F. Sanchez, Spectral-selective management of dissipative solitons in passive mode-locked fibre lasers, J. Opt. A: Pure Appl. Opt. 9 (2007) 1149-1156.

[29] N. Rebrova, T. Habruseva, G. Huyet, S.P. Hegarty, Stabilization of a passively mode-locked laser by continuous optical injection, Appl. Phys. Lett. 97 (2010) 101105.

[30] T. Habruseva, G. Huyet, S.P. Hegarty, Dynamics of quantum-dot mode-locked lasers with optical injection, IEEE J. Sel. Top. Quantum Electron. 17 (2011) $1272-1279$.

[31] A. Komarov, K. Komarov, D. Meshcheriakov, F. Amrani, F. Sanchez, Polarization dynamics in nonlinear anisotropic fibers, Phys. Rev. A 82 (2010) 013813.

[32] S. Chouli, Ph. Grelu, Rains of solitons in a fiber laser, Opt. Express 17 (2009) $11776-11781$.

[33] C. Bao, X. Xiao, C. Yang, Soliton rains in a normal dispersion fiber laser with dual-filter, Opt. Lett. 38 (2013) 1875-1877.

[34] A. Niang, F. Amrani, M. Salhi, Ph. Grelu, F. Sanchez, Rains of solitons in a figureof-eight passively mode-locked fiber laser, Appl. Phys. B (2014), http:// dx.doi.org/10.1007/s00340-014-5760-y.

[35] A. Niang, F. Amrani, M. Salhi, H. Leblond, A. Komarov, F. Sanchez, Harmonic mode-locking in a fiber laser through continuous external optical injection, Opt. Commun. 312 (2014) 1-6.
[36] K.P. Komarov, Theory of stationary ultrashort pulses in solid-state lasers with passive mode-locking, Opt. Spectrosc. 60 (1986) 231-234.

[37] H. Leblond, A. Niang, F. Amrani, M. Salhi, F. Sanchez, Motion of solitons of the complex Ginzburg-Landau equation: the effect of an external oscillating source, Phys. Rev. A 88 (2013) 033809.

[38] A. Komarov, K. Komarov, A. Niang, F. Sanchez, Nature of soliton interaction in fiber lasers with continuous external injection, Phys. Rev. A 89 (2014) 013833

[39] M. Horowitz, Y. Barad, Y. Silberberg, Noiselike pulses with a broadband spectrum generated from an erbium-doped fiber laser, Opt. Lett. 22 (1997) 799-801.

[40] M. Grapinet, Ph. Grelu, Vibrating soliton pairs in a mode-locked laser cavity, Opt. Lett. 31 (2006) 2115-2117.

[41] C. Lecaplain, Ph. Grelu, J.M. Soto-Crespo, N. Akhmediev, Dissipative rogue wave generation in multiple-pulsing mode-locked fiber laser, J. Opt. 15 (2013) 064005.

[42] J.M. Soto-Crespo, N. Akhmediev, G. Town, CW versus pulse regime in a passively mode-locked laser with a fast saturable absorber, J. Opt. Soc. Am. B 19 (2002) 234-242.

[43] A. Gordon, B. Fischer, Phase transition theory of many-mode ordering and pulse formation in lasers, Phys. Rev. Lett. 89 (2002) 103901.

[44] S. Chouli, Ph. Grelu, Soliton rains in a fiber laser: an experimental study, Phys Rev. A 81 (2010) 063829.

[45] J.P. Gordon, Dispersive perturbations of solitons of the nonlinear Schrödinger equation, J. Opt. Soc. Am. B 9 (1992) 91-97.

[46] S.S. Huang, Y.G. Wang, P.G. Yan, G.L. Zhang, J.Q. Zhao, H.Q. Li, R.Y. Lin, Soliton rains in a graphene-oxide passively mode-locked ytterbium-doped fiber laser with all-normal dispersion, Laser Phys. Lett. 11 (2014) 025102.

[47] C. Lecaplain, Ph. Grelu, Multi-gigahertz repetition-rate-selectable passive harmonic mode-locking of a fiber laser, Opt. Express 21 (2013) 10897-10902.

[48] M. Salhi, H. Leblond, F. Sanchez, High power tunable all-fiber double-clad Er:Yb:Silicate fiber laser, Opt. Commun. 247 (2005) 181-185.

[49] A. Komarov, K. Komarov, F. Sanchez, Quantization of binding energy of structural solitons in passive mode-locked fiber lasers, Phys. Rev. A 79 (2009) 033807.

[50] H.A. Haus, K. Tamura, L.E. Nelson, E.P. Ippen, Stretched-pulse additive pulse mode-locking in fiber ring lasers: theory and experiment, IEEE J. Quantun Electron. 31 (1995) 591-598.

[51] H. Leblond, M. Salhi, A. Hideur, T. Chartier, M. Brunel, F. Sanchez, Experimenta and theoretical study of the passively mode-locked Ytterbium-doped doubleclad fiber laser, Phys. Rev. A 65 (2002) 063811.

[52] A. Komarov, H. Leblond, F. Sanchez, Quintic complex Ginzburg-Landau model for ring fiber lasers, Phys. Rev. E 72 (2005) 025604(R).

53] E. Ding, J.N. Kutz, Operating regimes, split-step modeling, and the Haus master mode-locking model, J. Opt. Soc. Am. B 26 (2009) 2290-2300.

[54] M. Salhi, A. Haboucha, H. Leblond, F. Sanchez, Theoretical study of figure-eight all fiber laser, Phys. Rev. A 77 (2008) 033828.

[55] N. Akhmediev, J.M. Soto-Crespo, M. Grapinet, Ph. Grelu, Dissipative soliton interactions inside a fiber laser cavity, Opt. Fiber Technol. 11 (2005) 209-228.

[56] I.S. Aranson, L. Kramer, The world of the complex Ginzburg-Landau equation, Rev. Mod. Phys. 74 (2002) 99-143.

[57] B.A. Malomed, Bound solitons in the nonlinear Schrodinger-Ginzburg-Landau equation, Phys. Rev. A 44 (1991) 6954-6957.

58] V.V. Afanasjev, B.A. Malomed, P.L. Chu, Stability of bound states of pulses in the Ginzburg-Landau equations, Phys. Rev. E 56 (1997) 6020-6025.

[59] N.N. Akhmediev, A. Ankiewicz, J.M. Soto-Crespo, Multisoliton solutions of the complex Ginzburg-Landau equation, Phys. Rev. Lett. 79 (1997) 4047-4051.

[60] N.N. Akhmediev, A. Ankiewicz, J.M. Soto-Crespo, Stable soliton pairs in optica transmission lines and fiber lasers, J. Opt. Soc. Am. B 15 (1998) 515-523.

[61] H. Leblond, A. Komarov, M. Salhi, A. Haboucha, F. Sanchez, Cis bound states of three localized pulses of the cubic-quintic CGL equation, J. Opt. A: Pure Appl. Opt. 8 (2006) 319-326.

[62] A. Komarov, F. Amrani, A. Dmitriev, K. Komarov, D. Meshcheriakov, F. Sanchez Dispersive-wave mechanism of interaction between ultrashort pulses in passive mode-locked fiber lasers, Phys. Rev. A 85 (2012) 013802. 